\title{
Energy/Dissipation-Preserving Birkhoffian Multi-Symplectic Methods for Maxwell's Equations with Dissipation Terms
}

\author{
Hongling $\mathrm{Su}^{*}$ \\ Department of Mathematics, Renmin University of China, Beijing, 100872, China \\ Shengtai Li \\ Theoretical Division, Los Alamos National Lab, Los Alamos NM 87544, USA
}

\begin{abstract}
In this paper, we propose two new energy/dissipation-preserving Birkhoffian multi-symplectic methods (Birkhoffian and Birkhoffian box) for Maxwell's equations with dissipation terms. After investigating the non-autonomous and autonomous Birkhoffian formalism for Maxwell's equations with dissipation terms, we first apply a novel generating functional theory to the non-autonomous Birkhoffian formalism to propose our Birkhoffian scheme, and then implement a central box method to the autonomous Birkhoffian formalism to derive the Birkhoffian box scheme. We have obtained four formal local conservation laws and three formal energy global conservation laws. We have also proved that both of our derived schemes preserve the discrete version of the global/local conservation laws. Furthermore, the stability, dissipation and dispersion relations are also investigated for the schemes. Theoretical analysis shows that the schemes are unconditionally stable, dissipation-preserving for Maxwell's equations in a perfectly matched layer (PML) medium and have second order accuracy in both time and space. Numerical experiments for problems with exact theoretical results are given to demonstrate that the Birkhoffian multi-symplectic schemes are much more accurate in preserving energy than both the exponential finite-difference time-domain (FDTD) method and traditional Hamiltonian scheme. We also solve the electromagnetic pulse (EMP) propagation problem and the numerical results show that the Birkhoffian scheme recovers the magnitude of the current source and reaction history very well even after long time propagation.
\end{abstract}

Keywords: structure-preserving method, computational electromagnetics, Birkhoffian multi-symplectic method, energy/dissipation-preserving method, Hamiltonian scheme, Maxwell's equations with dissipation terms 


\section{Introduction}

Compared with conservative system of Maxwell's equations in a lossless medium, Maxwell's equations with dissipation terms in a medium including conductor loss have much more numerous applications, such as the high temperature plasma, the design of the CPU microelectronic field, etc.. In particular, Maxwell's equations considered in a perfectly matched layer (PML) medium, first defined in [1], play an important role in the PML technique. The various applications lead to the investigation on the construction of efficient numerical integrator for simulating Maxwell's equations with dissipation terms.

The well-known Yee's finite-difference time-domain (FDTD) method first proposed by Yee [2] has been widely used and developed in computational electromagnetic over the past few decades (see, e.g., [3, 4, 5, 6], and references therein ). However, Yee's scheme is only conditionally stable so that it may require very small temporal step-size and become computational costly for long time integration. Meanwhile, it has been widely recognized that the remarkable superiority of the Hamiltonian symplectic and multi-symplectic schemes, which are structure-preserving schemes or geometric integrators, is the stability of long time numerical simulation, when compared with the traditional schemes. In addition, the Hamiltonian schemes may also preserve conservation laws, dispersion relations, and so on. For instance, the multi-symplectic partitioned Runge-Kutta (MSPRK) method (see, e.g., [7, 8], and references therein ), which is one of the most important and popular class of Hamiltonian schemes, preserves quadratic conservation laws for autonomous Hamiltonian partial differential equations (PDEs). In references $[9,10,11,12]$, Hamiltonian symplectic/multi-symplectic schemes are constructed for Maxwell's equations in free space and energy-conserving properties of such structure-preserving schemes for Maxwell's equations are investigated.

However, the Hamiltonian schemes will lose their advantages when applied to Maxwell's equations with dissipation terms. Since the system of Maxwell's equations with dissipation terms is neither conservative system nor Hamiltonian system, it does not have Hamiltonian symplecticity and multi-symplecticity structures. Therefore, in order to construct geometric numerical schemes for Maxwell's equations with dissipation terms, the study on general geometric structure of Maxwell's equations is necessary.

\footnotetext{
${ }^{*}$ Corresponding author

Email addresses: hongling_su@ruc.edu.cn (Hongling Su), sli@lanl.gov (Shengtai Li)
} 
In this paper, we study the geometric structure of Maxwell's equations including conductor loss from four different formulations, which include non-autonomous Birkhoffian multisymplectic formulation, autonomous Birkhoffian multi-symplectic formulation, Hamiltonian multi-symplectic formulation and infinite dimensional Birkhoffian formulation. The nonautonomous Birkhoffian multi-symplectic formulation and the infinite dimensional Birkhoffian formulation expressed the original geometrical structures of the electromagnetic field, while the autonomous Birkhoffian formulation and the Hamiltonian formulation display the structures of the fake electromagnetic field transformed with an attenuation factor. Based on these formulations, we propose two Birkhoffian symplectic/multi-symplectic (BSMS) schemes: Birkhoffian scheme and Birkhoffian box scheme. We then investigate their theoretical and numerical behavior including their unconditionally stability, dispersion relations, dissipation and energy-preserving properties. Furthermore, we compare the behavior of the derived BSMS schemes and the central box scheme applied directly to Maxwell's equations in common formulation. The exponential Yee's scheme, which is often used in PML technique, is also compared. Both the central box scheme and the exponential Yee's scheme are Hamiltonian symplectic/muliti-symplectic scheme which have structure-preserving and quadratic energy-preserving properties for Hamiltonian systems. The comparison shows that, for Maxwell's equations in PML medium, the BSMS schemes not only preserve the geometric structures of Maxwell's equations, but also preserve the formal multi-symplectic energy/momentum conservation laws. Additionally, the BSMS schemes preserve exactly two formal quadratic conservation laws, while the two Hamiltonian schemes do not preserve any one of these conservation laws.

The outline of this paper is organized as follows: In section 2, we discuss the Birkhoffian and Hamiltonian structures for Maxwell's equations with dissipation terms. The formal multi-symplectic energy/momentum conservation laws are given. A formal quadratic conservation laws of Poynting energy for the case of $\sigma_{1}=\sigma_{2}$ are also derived along with a particular quadratic conservation law of $(\nabla \times \vec{E}, \nabla \times \vec{H})$. In section 3 , we introduce a new generating functional method to discretize in temporal direction the Maxwell's equation$\mathrm{s}$ in the non-autonomous Birkhoffian formulation, then a BSMS scheme is constructed by applying the mid-point method in spatial direction, we name it 'Birkhoffian scheme'. Applying the central box scheme to the autonomous Birkhoffian formulation, we obtain another BSMS scheme named 'Birkhoffian box scheme'. It is followed by the stability and dispersion relation discussions as well as the energy and dissipation-preserving discussions for the 
established schemes in section 4. In section 5, the numerical experiments are provided to compare the schemes. The paper is concluded and remarked in section 6 .

\section{Birkhoffian and Hamiltonian formulations for Maxwell's equations}

We consider a case without source field, then Maxwell's equations in medium involving conductor loss can be described as,

$$
\begin{aligned}
-\frac{\partial \vec{E}}{\partial t}+\frac{1}{\varepsilon} \nabla \times \vec{H}-\sigma_{1} \vec{E} & =0, \\
\frac{\partial \vec{H}}{\partial t}+\frac{1}{\mu} \nabla \times \vec{E}+\sigma_{2} \vec{H} & =0,
\end{aligned}
$$

where $\vec{H}$ and $\vec{E}$ are the magnetic and electric field intensities respectively, $\mu$ is the permeability, and $\varepsilon$ is the permittivity; $\sigma_{1}=\frac{\sigma}{\varepsilon}, \sigma_{2}=\frac{\sigma_{m}}{\mu}, \sigma$ and $\sigma_{m}$ are the electric and magnetic conductivities (dissipation factors).

\subsection{Non-autonomous Birkhoffian multi-symplectic formulation}

Left-multiplying $e^{\left(\sigma_{1}+\sigma_{2}\right) t}$ to (1), we have the following non-autonomous Birkhoffian multisymplectic formulation

$$
M(t) u_{t}+K_{1}(t) u_{x}+K_{2}(t) u_{y}+K_{3}(t) u_{z}=\nabla B(u, t)+\frac{\partial F(u, t)}{\partial t},
$$

where $u=\left(\vec{H}^{\top}, \vec{E}^{\top}\right)^{\top}$, the Birkhoffian functions $B(u, t)$ and $F(u, t)$ are defined as

$$
B=\frac{\sigma_{1}-\sigma_{2}}{2} e^{\left(\sigma_{1}+\sigma_{2}\right) t} \vec{E} \cdot \vec{H}, \quad F=\frac{1}{2} e^{\left(\sigma_{1}+\sigma_{2}\right) t}\left(\vec{E}^{\top},-\vec{H}^{\top}\right)^{\top},
$$

$M(t)$ and $K_{i}(t)$ are skew-symmetric matrices,

$$
M(t)=e^{\left(\sigma_{1}+\sigma_{2}\right) t}\left(\begin{array}{cc}
0 & -I_{3 \times 3} \\
I_{3 \times 3} & 0
\end{array}\right), \quad K_{i}(t)=e^{\left(\sigma_{1}+\sigma_{2}\right) t}\left(\begin{array}{cc}
\frac{1}{\varepsilon} A_{i} & 0 \\
0 & \frac{1}{\mu} A_{i}
\end{array}\right),
$$

and

$$
A_{1}=\left(\begin{array}{ccc}
0 & 0 & 0 \\
0 & 0 & -1 \\
0 & 1 & 0
\end{array}\right), \quad A_{2}=\left(\begin{array}{ccc}
0 & 0 & 1 \\
0 & 0 & 0 \\
-1 & 0 & 0
\end{array}\right), \quad A_{3}=\left(\begin{array}{ccc}
0 & -1 & 0 \\
1 & 0 & 0 \\
0 & 0 & 0
\end{array}\right)
$$

Birkhoffian multi-symplectic formulation, also called Birkhoffian PDEs, is a generalization of Hamiltonian PDEs. The key difference between Birkhoffian PDEs and Hamiltonian PDEs 
is that the skew-symmetric matrices $M$ and $K_{i}$ in Birkhoffian formulation depend on independent variable $t$, hence it results in the dissipation terms $\frac{\partial F}{\partial t}$ in Eq. (2). For more details of Birkhoffian PDEs theory, please refer to [13].

Theorem 2.1.1. (Birkhoffian multi-symplecticity conservation) If $\left(d \vec{H}^{\top}, d \vec{E}^{\top}\right)^{\top}$ is the solution of the variational equations associated with (2), then it holds the following multisymplectic conservation law

$$
\frac{\partial}{\partial t}\left(e^{\left(\sigma_{1}+\sigma_{2}\right) t} d \vec{E} \wedge d \vec{H}\right)+e^{\left(\sigma_{1}+\sigma_{2}\right) t} \sum_{i=1}^{3} \frac{\partial}{\partial X_{i}}\left(\frac{1}{\varepsilon} d \vec{H} \wedge A_{i} d \vec{H}+\frac{1}{\mu} d \vec{E} \wedge A_{i} d \vec{E}\right)=0,
$$

where $X_{1}=x, X_{2}=y, X_{3}=z$.

This multi-symplectic conservation law can be derived from a result about multi-symplecticity of Birkhoffian PDEs (cf. [13]), thereby the proof is omitted here.

If $\sigma_{1}=\sigma_{2}=\bar{\sigma}$, Birkhoffian formulation (2) can be rewritten as

$$
\left(\begin{array}{cc}
0 & -e^{2 \bar{\sigma} t} \\
e^{2 \bar{\sigma} t} & 0
\end{array}\right)\left(\begin{array}{c}
\vec{H}_{t} \\
\vec{E}_{t}
\end{array}\right)+\left(\begin{array}{cc}
\frac{e^{2 \bar{\sigma} t}}{\varepsilon} \nabla \times & 0 \\
0 & \frac{e^{2 \bar{\sigma} t}}{\mu} \nabla \times
\end{array}\right)\left(\begin{array}{c}
\vec{H} \\
\vec{E}
\end{array}\right)=\left(\begin{array}{c}
\bar{\sigma} e^{2 \bar{\sigma} t} \vec{E} \\
-\bar{\sigma} e^{2 \bar{\sigma} t} \vec{H}
\end{array}\right),
$$

which deduce formal energy local and global conservation laws besides the multi-symplectic conservation law (6).

Theorem 2.1.2. (Conservation I) If $\vec{H}$ and $\vec{E}$ are the solutions of the Maxwell's equations (1) in medium with $\sigma_{1}=\sigma_{2}=\bar{\sigma}$, then it holds a formal energy local conservation law as

$$
\frac{\partial}{\partial t}\left[e^{2 \bar{\sigma} t}\left(\frac{\varepsilon}{2}|\vec{E}|^{2}+\frac{\mu}{2}|\vec{H}|^{2}\right)\right]+e^{2 \bar{\sigma} t} \nabla \cdot(\vec{E} \times \vec{H})=0 .
$$

If the solution $(\vec{H}, \vec{E})$ satisfies periodic boundary condition or the following boundary conditions:

$$
\vec{E} \times \vec{n}=0, \quad \text { or } \quad \vec{H} \times \vec{n}=0,
$$

then it holds the following formal energy global conservation law

$$
\frac{d \mathcal{H}_{1}}{d t}=0, \quad \mathcal{H}_{1}=\int_{X} e^{2 \bar{\sigma} t}\left(\varepsilon|\vec{E}|^{2}+\mu|\vec{H}|^{2}\right) d X
$$

which means that the electromagnetic energy in the Poynting's theorem dissipates exponentially along time. 
Proof. Left-multiplying $\left(-\varepsilon \vec{E}^{\top}, \mu \vec{H}^{\top}\right)^{\top}$ to (7), we have

$$
\frac{\partial}{\partial t}\left[e^{2 \bar{\sigma} t}\left(\frac{\varepsilon}{2}|\vec{E}|^{2}+\frac{\mu}{2}|\vec{H}|^{2}\right)\right]+e^{2 \bar{\sigma} t}(-\nabla \times \vec{H} \cdot \vec{E}+\vec{H} \cdot \nabla \times \vec{E})=0,
$$

note that $-\nabla \times \vec{H} \cdot \vec{E}+\vec{H} \cdot \nabla \times \vec{E}=\nabla \cdot(\vec{E} \times \vec{H})$, we obtain (8). Integrating (8) with respect to space $X=(x, y, z)$, we get

$$
\frac{d \mathcal{H}_{1}}{d t}+e^{2 \bar{\sigma} t} \int_{X} \nabla \cdot(\vec{E} \times \vec{H}) d X=0 .
$$

Using Gauss' law and by periodic boundary condition or the boundary conditions (9), we have

$$
\int_{X} \nabla \cdot(\vec{E} \times \vec{H}) d X=\int_{\partial X} \vec{E} \times \vec{H} \cdot \vec{n} d S=0
$$

This completes the proof of the theorem.

Similarly, left-multiplying $\left(\nabla \times \vec{H}_{t}, \nabla \times \vec{E}_{t}\right)$ to $(7)$ yields another local conservation law as following:

$$
\frac{\partial}{\partial t}\left[e^{2 \bar{\sigma} t}\left(\frac{1}{2 \varepsilon}|\nabla \times \vec{H}|^{2}+\frac{1}{2 \mu}|\nabla \times \vec{E}|^{2}\right)\right]+e^{2 \bar{\sigma} t} \nabla \cdot\left[\left(\bar{\sigma} \vec{E}+\vec{E}_{t}\right) \times\left(\bar{\sigma} \vec{H}+\vec{H}_{t}\right)\right]=0,
$$

which results in a special quadratic global conservation law, that is,

Theorem 2.1.3. (Conservation II) If $\vec{H}$ and $\vec{E}$ are the solutions of the Maxwell's equations (1) in medium with $\sigma_{1}=\sigma_{2}=\bar{\sigma}$, then (14) is preserved. If the solution satisfies periodic boundary condition or the boundary conditions (9), then it preserves the following quadratic conservation law:

$$
\frac{d \mathcal{H}_{2}}{d t}=0, \quad \mathcal{H}_{2}=\int_{X} e^{2 \bar{\sigma} t}\left(\frac{1}{\varepsilon}|\nabla \times \vec{H}|^{2}+\frac{1}{\mu}|\nabla \times \vec{E}|^{2}\right) d X
$$

We note that $\vec{E}_{t} \times \vec{n}=0$ if $\vec{E} \times \vec{n}=0$, and the same result holds for $\vec{H}$, therefore the proof of this theorem is similar to that of the theorem 2.1.2 and is omitted here.

Introducing the helicities (cf. [14]) of $\vec{E}$ and $\vec{H}$, which are defined as

$$
\hbar(\vec{E}, t)=\int_{X} \vec{E} \cdot \nabla \times \vec{E} \mathrm{~d} X, \quad \hbar(\vec{H}, t)=\int_{X} \vec{H} \cdot \nabla \times \vec{H} \mathrm{~d} X,
$$

and left-multiplying $\left(\vec{H}_{t}, \vec{E}_{t}\right)$ to $(7)$, we obtain

$$
\frac{\partial}{\partial t}\left[e^{2 \bar{\sigma} t}\left(\frac{1}{\varepsilon} \vec{H} \cdot \nabla \times \vec{H}+\frac{1}{\mu} \vec{E} \cdot \nabla \times \vec{E}\right)\right]+e^{2 \bar{\sigma} t} \nabla \cdot\left[\vec{H} \times\left(\bar{\sigma} \vec{H}+\vec{H}_{t}\right)+\vec{E} \times\left(\bar{\sigma} \vec{E}+\vec{E}_{t}\right)\right]=0,(17
$$


which is called multi-symplectic energy conservation law following the terminology used in Hamiltonian framework. This results in a global conservation law of the helicity with the dissipation factor:

Theorem 2.1.4. (Conservation III) If $\vec{H}$ and $\vec{E}$ are the solutions of the Maxwell's equations (1) with periodic boundary condition in medium with $\sigma_{1}=\sigma_{2}=\bar{\sigma}$, then it holds

$$
\frac{d \mathcal{H}_{3}}{d t}=0, \quad \mathcal{H}_{3}=e^{2 \bar{\sigma} t}\left(\frac{1}{\varepsilon} \hbar(\vec{H}, t)+\frac{1}{\mu} \hbar(\vec{E}, t)\right) .
$$

The proof is omitted.

Moreover, left-multiplying $\left(\partial_{x_{i}} \vec{H}, \partial_{x_{i}} \vec{E}\right)$ to (7) leads to three multi-symplectic momentum conservation laws. For simplicity, we only give one of them for the case $x_{1}=x$,

$$
\begin{array}{r}
\frac{\partial}{\partial t}\left[e^{2 \bar{\sigma} t}\left(\vec{E}_{x} \cdot \vec{H}-\vec{H}_{x} \cdot \vec{E}\right)\right]+\frac{\partial}{\partial x}\left[e^{2 \bar{\sigma} t}\left(\vec{H}_{t} \cdot \vec{E}-\vec{E}_{t} \cdot \vec{H}\right)\right] \\
+\frac{\partial}{\partial x}\left[e^{2 \bar{\sigma} t}\left(\frac{1}{\varepsilon} \vec{H} \cdot\left(A_{2} \vec{H}_{y}+A_{3} \vec{H}_{z}\right)+\frac{1}{\mu} \vec{E} \cdot\left(A_{2} \vec{E}_{y}+A_{3} \vec{E}_{z}\right)\right)\right] \\
+\frac{\partial}{\partial y}\left[e^{2 \bar{\sigma} t}\left(\frac{1}{\varepsilon} \vec{H}_{x} \cdot A_{2} \vec{H}+\frac{1}{\mu} \vec{E}_{x} \cdot A_{2} \vec{E}\right)\right] \\
+\frac{\partial}{\partial z}\left[e^{2 \bar{\sigma} t}\left(\frac{1}{\varepsilon} \vec{H}_{x} \cdot A_{3} \vec{H}+\frac{1}{\mu} \vec{E}_{x} \cdot A_{3} \vec{E}\right)\right]=0 .
\end{array}
$$

\subsection{Autonomous Birkhoffian multi-symplectic formulation}

Suppose $\varepsilon$ and $\mu$ are space-independent, we introduce the following transformation

$$
\vec{E}=e^{-\sigma_{1} t} E, \quad \vec{H}=e^{-\sigma_{2} t} H,
$$

then the equations (1) can be rewritten as

$$
\begin{gathered}
-\frac{\partial E}{\partial t}+\frac{1}{\varepsilon_{1}(t)} \nabla \times H=0, \\
\frac{\partial H}{\partial t}+\frac{1}{\mu_{1}(t)} \nabla \times E=0,
\end{gathered}
$$

where $\varepsilon_{1}(t)=\varepsilon e^{\left(\sigma_{2}-\sigma_{1}\right) t}$ and $\mu_{1}(t)=\mu e^{\left(\sigma_{1}-\sigma_{2}\right) t}$. The above system has an autonomous Birkhoffian multi-symplectic formulation as

$$
\bar{M} u_{t}+\bar{K}_{1}(t) u_{x}+\bar{K}_{2}(t) u_{y}+\bar{K}_{3}(t) u_{z}=\nabla \bar{S}(u, t)
$$

where $u=\left(H^{\top}, E^{\top}\right)^{\top}$ and

$$
\bar{S}=0, \quad \bar{M}=\left(\begin{array}{cc}
0 & -I_{3 \times 3} \\
I_{3 \times 3} & 0
\end{array}\right), \quad \bar{K}_{i}=\left(\begin{array}{cc}
\frac{1}{\varepsilon_{1}(t)} A_{i} & 0 \\
0 & \frac{1}{\mu_{1}(t)} A_{i}
\end{array}\right), \quad i=1,2,3 .
$$


The multi-symplectic formulation (22) preserves the following multi-symplectic conservation law

$$
\frac{\partial}{\partial t}(d E \wedge d H)+\sum_{i=1}^{3} \frac{\partial}{\partial X_{i}}\left(\frac{1}{\varepsilon_{1}(t)} d H \wedge A_{i} d H+\frac{1}{\mu_{1}(t)} d E \wedge A_{i} d E\right)=0,
$$

which reduces to the multi-symplectic conservation law (6) via the inverse transformation of $(20)$.

If $\sigma_{1}=\sigma_{2}=\bar{\sigma}$, then the (22) is autonomous Hamiltonian multi-symplectic PDEs, it is well-known that the autonomous Hamiltonian PDEs have the general expressions for the multi-symplectic energy/momentum conservation laws, we refer the readers to $[15,16,17]$ for more details. In this case, we have a multi-symplectic energy conservation law:

$$
\frac{\partial}{\partial t}\left(\frac{1}{\varepsilon} H \cdot \nabla \times H+\frac{1}{\mu} E \cdot \nabla \times E\right)-\sum_{i=1}^{3} \frac{\partial}{\partial X_{i}}\left(\frac{1}{\varepsilon} H \cdot A_{i} H_{t}+\frac{1}{\mu} E \cdot A_{i} E_{t}\right)=0,
$$

and three multi-symplectic momentum conservation laws:

$$
\begin{aligned}
& \frac{\partial}{\partial t}\left(-\partial_{X_{i}} H \cdot E+\partial_{X_{i}} E \cdot H\right)+\frac{\partial}{\partial X_{i}}\left(H_{t} \cdot E-E_{t} \cdot H\right) \\
+ & \sum_{j=1, j \neq i}^{3} \frac{\partial}{\partial X_{j}}\left(\frac{1}{\varepsilon} \partial_{X_{i}} H \cdot A_{j} H+\frac{1}{\mu} \partial_{X_{i}} E \cdot A_{j} E\right) \\
+ & \frac{\partial}{\partial X_{i}} \sum_{j=1, j \neq i}^{3}\left(\frac{1}{\varepsilon} H \cdot A_{j} \partial_{X_{j}} H+\frac{1}{\mu} E \cdot A_{j} \partial_{X_{j}} E\right)=0, \quad i=1,2,3 .
\end{aligned}
$$

We also obtain a local quadratic conservation law equivalent to (8) as

$$
\frac{\partial}{\partial t}\left(\frac{\varepsilon}{2}|E|^{2}+\frac{\mu}{2}|H|^{2}\right)+\nabla \cdot(E \times H)=0
$$

and a conservation law equivalent to (14), it reads

$$
\frac{\partial}{\partial t}\left(\frac{1}{2 \varepsilon}|\nabla \times H|^{2}+\frac{1}{2 \mu}|\nabla \times E|^{2}\right)+\nabla \cdot\left(H_{t} \times E_{t}\right)=0 .
$$

Applying the inverse transformation of (20) to (25)-(28), we can recover the local conservation laws (17), (19), (8) and (14).

Given appropriate boundary conditions such as $E \times \vec{n}=0$ or $H \times \vec{n}$ or periodic boundary conditions, by direct integrating (25), (27) and (28), respectively, we obtain the following global conservation laws:

$$
\frac{\mathrm{d}}{\mathrm{d} t} \int_{X}\left(\frac{1}{\varepsilon} H \cdot \nabla \times H+\frac{1}{\mu} E \cdot \nabla \times E\right) d X=0 .
$$




$$
\begin{gathered}
\frac{\mathrm{d}}{\mathrm{d} t} \int_{X}\left(\varepsilon|E|^{2}+\mu|H|^{2}\right) d X=0, \\
\frac{\mathrm{d}}{\mathrm{d} t} \int_{X}\left(\frac{1}{\varepsilon}|\nabla \times H|^{2}+\frac{1}{\mu}|\nabla \times E|^{2}\right) d X=0,
\end{gathered}
$$

which reduce respectively to the conservation laws (18), (10) and (15).

If there is no conductor loss in the medium, i.e. $\bar{\sigma}=0$, then $\vec{E}=E, \vec{H}=H$, and the Maxwell's equations (1) has a standard multi-symplectic Hamiltonian formulation discussed in [9]. By 'standard' we refer to the fact that both the skew-symmetric matrices $\bar{M}$ and $\bar{K}_{i}$ are constant matrix. Consequently the derived multi-symplectic energy and momentum conservation laws reduce to the conservation laws discussed in $[10,11,18,19]$.

Remark 2.2.1. $\sigma_{1}=\sigma_{2}=\bar{\sigma} \neq 0$ implies that the impedance of the medium with conductor loss matches that of free space, perhaps not always met in practical computations, but it is the key condition of PML layer for solving unbounded electromagnetic problems. Therefore, the investigation of numerical methods for Maxwell's equations in such special case is also very important.

Remark 2.2.2. Although the non-autonomous Birkhoffian formulation and its related conservation laws can be recovered via transformation from the autonomous Birkhoffian formulation, the numerical schemes for the two formulations and their effectiveness in preserving the conservation laws numerically, which will be discussed in more detail in Section 3 to 5, are quite different.

\subsection{Hamiltonian multi-symplectic formulation}

Consider the following transformations:

$$
\vec{H}=e^{-\frac{\sigma_{1}+\sigma_{2}}{2} t} H, \quad \vec{E}=e^{-\frac{\sigma_{1}+\sigma_{2}}{2} t} E
$$

then Eqs (1) becomes

$$
\begin{aligned}
-\frac{\partial E}{\partial t}+\frac{1}{\varepsilon} \nabla \times H & =\frac{\sigma_{1}-\sigma_{2}}{2} E, \\
\frac{\partial H}{\partial t}+\frac{1}{\mu} \nabla \times E & =\frac{\sigma_{1}-\sigma_{2}}{2} H,
\end{aligned}
$$

which has a Hamiltonian multi-symplectic formulation as

$$
\tilde{M} u_{t}+\tilde{K}_{1} u_{x}+\tilde{K}_{2} u_{y}+\tilde{K}_{3} u_{z}=\nabla \tilde{S}(u, t),
$$


where $u=\left(H^{\top}, E^{\top}\right)^{\top}$ and

$$
\tilde{M}=\left(\begin{array}{cc}
0 & -I_{3 \times 3} \\
I_{3 \times 3} & 0
\end{array}\right), \quad \tilde{K}_{i}=\left(\begin{array}{cc}
\frac{1}{\varepsilon} A_{i} & 0 \\
0 & \frac{1}{\mu} A_{i}
\end{array}\right), \quad i=1,2,3,
$$

and

$$
\widetilde{S}=\frac{\sigma_{1}-\sigma_{2}}{2} H \cdot E
$$

Remark 2.3.1. Since $H \perp E$, i.e. $\tilde{S}=0$, therefore we consider the above formula of $\tilde{S}$ only from the point of mathematics.

The formulation (34) is autonomous Hamiltonian PDEs, and it can recover all the conservation laws discussed previously. Furthermore, the formulation (34) is an autonomous system for any constant $\sigma_{1}$ and $\sigma_{2}$, therefore it has multi-symplectic energy and momentum conservation laws even if $\sigma_{1} \neq \sigma_{2}$. The multi-symplectic energy conservation law of (34) reads

$$
\begin{gathered}
\frac{\partial}{\partial t}\left(\frac{1}{2 \varepsilon} H \cdot \nabla \times H+\frac{1}{2 \mu} E \cdot \nabla \times E+\frac{\sigma_{2}-\sigma_{1}}{2} H \cdot E\right)+\frac{\partial}{\partial x}\left(\frac{1}{2 \varepsilon} H_{t} \cdot A_{1} H+\frac{1}{2 \mu} E_{t} \cdot A_{1} E\right) \\
+\frac{\partial}{\partial y}\left(\frac{1}{2 \varepsilon} H_{t} \cdot A_{2} H+\frac{1}{2 \mu} E_{t} \cdot A_{2} E\right)+\frac{\partial}{\partial z}\left(\frac{1}{2 \varepsilon} H_{t} \cdot A_{3} H+\frac{1}{2 \mu} E_{t} \cdot A_{3} E\right)=0 .
\end{gathered}
$$

Using the inverse transform of (32), the above conservation law is transformed into the following form:

$$
\begin{aligned}
\frac{\partial}{\partial t}\left[e ^ { ( \sigma _ { 1 } + \sigma _ { 2 } ) t } \left(\frac{1}{\varepsilon} \vec{H}\right.\right. & \left.\left.\cdot \nabla \times \vec{H}+\frac{1}{\mu} \vec{E} \cdot \nabla \times \vec{E}+\left(\sigma_{2}-\sigma_{1}\right) \vec{H} \cdot \vec{E}\right)\right] \\
+ & \frac{\partial}{\partial x}\left[e^{\left(\sigma_{1}+\sigma_{2}\right) t}\left(\frac{1}{\varepsilon} \vec{H}_{t} \cdot A_{1} \vec{H}+\frac{1}{\mu} \vec{E}_{t} \cdot A_{1} \vec{E}\right)\right] \\
+ & \frac{\partial}{\partial y}\left[e^{\left(\sigma_{1}+\sigma_{2}\right) t}\left(\frac{1}{\varepsilon} \vec{H}_{t} \cdot A_{2} \vec{H}+\frac{1}{\mu} \vec{E}_{t} \cdot A_{2} \vec{E}\right)\right] \\
+\frac{\partial}{\partial z} & {\left[e^{\left(\sigma_{1}+\sigma_{2}\right) t}\left(\frac{1}{\varepsilon} \vec{H}_{t} \cdot A_{3} \vec{H}+\frac{1}{\mu} \vec{E}_{t} \cdot A_{3} \vec{E}\right)\right]=0, }
\end{aligned}
$$

which reduces to the conservation law (17) when $\sigma_{1}=\sigma_{2}$. One of the three multi-symplectic momentum conservation law is

$$
\begin{array}{r}
\frac{\partial}{\partial t}\left(\partial_{x} E \cdot H-\partial_{x} H \cdot E\right)+\frac{\partial}{\partial x}\left(H_{t} \cdot E-E_{t} \cdot H+\left(\sigma_{2}-\sigma_{1}\right) H \cdot E\right) \\
+\frac{\partial}{\partial x}\left(\frac{1}{\varepsilon}\left(H \cdot A_{2} \partial_{y} H+H \cdot A_{3} \partial_{z} H\right)+\frac{1}{\mu}\left(E \cdot A_{2} \partial_{y} E+E \cdot A_{3} \partial_{z} E\right)\right) \\
+\frac{\partial}{\partial y}\left(\frac{1}{\varepsilon} \partial_{x} H \cdot A_{2} H+\frac{1}{\mu} \partial_{x} E \cdot A_{2} E\right)+\frac{\partial}{\partial z}\left(\frac{1}{\varepsilon} \partial_{x} H \cdot A_{3} H+\frac{1}{\mu} \partial_{x} E \cdot A_{3} E\right)=0,
\end{array}
$$


which can be rewritten in

$$
\begin{array}{r}
\frac{\partial}{\partial t}\left[e^{\left(\sigma_{1}+\sigma_{2}\right) t}\left(\partial_{x} \vec{E} \cdot \vec{H}-\partial_{x} \vec{H} \cdot \vec{E}\right)\right]+ \\
+\frac{\partial}{\partial x}\left[e^{\left(\sigma_{1}+\sigma_{2}\right) t}\left(\frac{1}{\varepsilon} \vec{H} \cdot\left(A_{2} \partial_{y} \vec{H}+A_{3} \partial_{z} \vec{H}\right)+\frac{1}{\mu} \vec{E} \cdot\left(A_{2} \partial_{y} \vec{E}+A_{3} \partial_{z} \vec{E}\right)\right)\right] \\
+\frac{\partial}{\partial y}\left[e^{\left(\sigma_{1}+\sigma_{2}\right) t}\left(\frac{1}{\varepsilon} \partial_{x} \vec{H} \cdot A_{2} \vec{H}+\frac{1}{\mu} \partial_{x} \vec{E} \cdot A_{2} \vec{E}\right)\right] \\
+\frac{\partial}{\partial z}\left[e^{\left(\sigma_{1}+\sigma_{2}\right) t}\left(\frac{1}{\varepsilon} \partial_{x} \vec{H} \cdot A_{3} \vec{H}+\frac{1}{\mu} \partial_{x} \vec{E} \cdot A_{3} \vec{E}\right)\right]=0
\end{array}
$$

Obviously, this reduces to (19) when $\sigma_{1}=\sigma_{2}$. We also obtain a general conservation law of the helicity,

$$
\frac{d}{d t} \int_{X} e^{\left(\sigma_{1}+\sigma_{2}\right) t}\left(\frac{1}{\varepsilon} \vec{H} \cdot \nabla \times \vec{H}+\frac{1}{\mu} \vec{E} \cdot \nabla \times \vec{E}+\frac{\sigma_{2}-\sigma_{1}}{2} \vec{H} \cdot \vec{E}\right) d X=0,
$$

which reduces to the helicity conservation law (18) if $\sigma_{1}=\sigma_{2}$.

Remark 2.3.2. Based on the discussions in subsection 2.1-2.3, we conclude that the Maxwell's equations (1) preserves the multi-symplectic energy and momentum conservation laws (38) and (40), and preserves the global conservation law (41). When $\sigma_{1}=\sigma_{2}$, it also preserves the local conservation laws (8) and (14), and, with appropriate boundary conditions, it preserves the global energy conservation law (10) and (15).

\subsection{Infinite dimensional Birkhoffian symplectic formulation}

The formulation (2) can be rewritten into an infinite dimensional Birkhoffian symplectic formulation as following,

$$
M(t) u_{t}=\delta \mathcal{B}(u)+\frac{\partial F(u, t)}{\partial t},
$$

with the matrix $M(t)$ in (4) and vector function $F(u, t)$ in (3), and the functional

$$
\mathcal{B}(z)=\mathcal{B}(\vec{H}, \vec{E})=\int_{X} e^{\left(\sigma_{1}+\sigma_{2}\right) t}\left(\frac{\sigma_{1}-\sigma_{2}}{2} \vec{E} \cdot \vec{H}-\frac{1}{2 \varepsilon} \vec{H} \cdot \nabla \times \vec{H}-\frac{1}{2 \mu} \vec{E} \cdot \nabla \times \vec{E}\right) d X .
$$

We assume the initial electromagnetic field as $\left(\vec{H}_{0}, \vec{E}_{0}\right)$ at $t=0$, and if the electromagnetic field satisfies periodic boundary condition, then the field preserves an infinite dimensional two-form as

$$
\int_{X} e^{\left(\sigma_{1}+\sigma_{2}\right) t} \delta \vec{E} \wedge \delta \vec{H}=\int_{X} \delta \vec{E}_{0} \wedge \delta \vec{H}_{0} .
$$

From this infinite dimensional formulation the global energy conservation laws (10), (15) and (18) can be recovered. 


\section{Numerical Discretizations}

In this section we use a generating functional theory to establish the Birkhoffian scheme for the Maxwell's equations in non-autonomous Birkhoffian formulation in subsection 3.1. In subsection 3.2 we apply the central box method to the Maxwell's equations in autonomous Birkhoffian formulation to achieve the Birkhoffian box scheme. In subsection 3.3, we implement the central box method to the Maxwell's equations in Hamiltonian formulation. Furthermore, we discuss the relationship between the Birkhoffian scheme and the central box method. Since the multi-symplecticity and the energy/momentumpreserving property of the central box method has been discussed in many references (e.g. $[15,17,7,8,21,22,23,24])$, we can investigate the energy-preserving properties of the Birkhoffian scheme easily, which will be done in section 4 .

At first, we recall a brief definition of Birkhoffian multi-symplectic algorithm. Given a Birkhoffian PDEs

$$
M(u, x, t) u_{t}+K(u, x, t) u_{x}=\nabla B(u, x, t)+\frac{\partial F(u, x, t)}{\partial t}+\frac{\partial G(u, x, t)}{\partial x},
$$

which has a Birkhoffian multi-symplectic conservation law as

$$
\partial_{t}(M(u, x, t) d u \wedge d u)+\partial_{x}(K(u, x, t) d u \wedge d u)=0 .
$$

Applying a discrete method to (45), we get

$$
\begin{aligned}
M\left(u_{i}^{j}, x_{i}, t_{j}\right)\left[\partial_{t}\right]_{i}^{j} u_{i}^{j}+K\left(u_{i}^{j}, x_{i}, t_{j}\right)\left[\partial_{x}\right]_{i}^{j} u_{i}^{j} & =\left(\nabla B\left(u_{i}^{j}, x_{i}, t_{j}\right)\right)_{i}^{j}+\left(\partial_{t} F\left(u_{i}^{j}, x_{i}, t_{j}\right)\right)_{i}^{j} \\
& +\left(\partial_{x} G\left(u_{i}^{j}, x_{i}, t_{j}\right)\right)_{i}^{j},
\end{aligned}
$$

where $u_{i}^{j} \approx u\left(x_{i}, t_{j}\right), i$ is the spatial index and $j$ temporal index in the discrete system. $\left[\partial_{x}\right]_{i}^{j}$ and $\left[\partial_{t}\right]_{i}^{j}$ denote the discretization of $\frac{\partial}{\partial x}$ and $\frac{\partial}{\partial t}$.

Definition 3.0.1. The numerical scheme determined by (47) is called a Birkhoffian multisymplectic scheme if it preserves the discrete multi-symplectic conservation law as

$$
\left[\partial_{t}\right]_{i}^{j}\left(M\left(u_{i}^{j}, x_{i}, t_{j}\right) d u_{i}^{j} \wedge d u_{i}^{j}\right)+\left[\partial_{x}\right]_{i}^{j}\left(K\left(u_{i}^{j}, x_{i}, t_{j}\right) d u_{i}^{j} \wedge d u_{i}^{j}\right)=0 .
$$

\subsection{A novel Birkhoffian scheme applied to non-autonomous Birkhoffian formulation}

The non-autonomous Birkhoffian system (2) with the infinite dimensional Birkhoffian formulation (42) preserves the symplectic conservation law (44). According to the generating functional theory developed in our work [25], it implies that there exists a functional 
$\mathcal{S}(v, w, t)$ such that

$$
\frac{\delta \mathcal{S}}{\delta v}=-e^{\frac{\left(\sigma_{1}+\sigma_{2}\right) t}{2}} \vec{E}+\vec{E}_{0}, \quad-\frac{\delta \mathcal{S}}{\delta w}=-e^{\frac{\left(\sigma_{1}+\sigma_{2}\right) t}{2}} \vec{H}+\vec{H}_{0},
$$

where

$$
2 w=e^{\frac{\left(\sigma_{1}+\sigma_{2}\right) t}{2}} \vec{E}+\vec{E}_{0}, \quad 2 v=e^{\frac{\left(\sigma_{1}+\sigma_{2}\right) t}{2}} \vec{H}+\vec{H}_{0}
$$

If the periodic boundary condition is satisfied, the mapping $\left(\vec{H}_{0}, \vec{E}_{0}\right) \mapsto(\vec{H}, \vec{E})$ determined by (49) preserves the conservation law (44). In what follows, we assume that periodic boundary condition is satisfied unless specified otherwise.

Theorem 3.1.1. If $\mathcal{S}(v, w, t)$ is a smooth solution of the generalized Hamiltonian-Jacobi equation:

$$
\frac{\partial \mathcal{S}}{\partial t}=\mathcal{B}\left(e^{-\left(\sigma_{1}+\sigma_{2}\right) t}\left(v+\frac{1}{2} \frac{\delta \mathcal{S}}{\delta w}\right), e^{-\left(\sigma_{1}+\sigma_{2}\right) t}\left(w-\frac{1}{2} \frac{\delta \mathcal{S}}{\delta v}\right), t\right)
$$

with initial value $\mathcal{S}(v, w, 0)=0$, and if the matrix

$$
\left(\begin{array}{cc}
I+\frac{1}{2} \frac{\delta^{2} \mathcal{S}}{\delta v \delta w} & -\frac{1}{2} \frac{\delta^{2} \mathcal{S}}{\delta v^{2}} \\
\frac{1}{2} \frac{\delta^{2} \mathcal{S}}{\delta w^{2}} & I-\frac{1}{2} \frac{\delta^{2} \mathcal{S}}{\delta w \delta v}
\end{array}\right)
$$

is invertible, then the mapping $\left(\vec{H}_{0}, \vec{E}_{0}\right) \mapsto(\vec{H}, \vec{E})$ determined by $(49)$ is the solution of Maxwell's equations with the formulation (42).

Proof. subtracting (49) from (50), we obtain,

$$
\vec{H}=e^{-\frac{\sigma_{1}+\sigma_{2}}{2} t}\left(v+\frac{1}{2} \frac{\delta \mathcal{S}}{\delta w}\right), \quad \vec{E}=e^{-\frac{\sigma_{1}+\sigma_{2}}{2} t}\left(w-\frac{1}{2} \frac{\delta \mathcal{S}}{\delta v}\right),
$$

Let $\frac{\delta \vec{H}}{\delta v}$ denote $\frac{\delta\left(\int_{x} \vec{H} d x\right)}{\delta v}$, then we have

$$
\left(\begin{array}{ll}
\left(\frac{\delta \vec{H}}{\delta v}\right)^{\top} & \left(\frac{\delta \vec{E}}{\delta v}\right)^{\top} \\
\left(\frac{\delta \vec{H}}{\delta w}\right)^{\top} & \left(\frac{\delta \vec{E}}{\delta w}\right)^{\top}
\end{array}\right)=e^{-\frac{\sigma_{1}+\sigma_{2}}{2} t}\left(\begin{array}{cc}
I+\frac{1}{2} \frac{\delta^{2} \mathcal{S}}{\delta w \delta v} & -\frac{1}{2} \frac{\delta^{2} \mathcal{S}}{\delta v^{2}} \\
\frac{1}{2} \frac{\delta^{2} \mathcal{S}}{\delta w^{2}} & I-\frac{1}{2} \frac{\delta^{2} \mathcal{S}}{\delta v \delta w}
\end{array}\right) .
$$

Calculating the variational derivatives of the equation (51) with respect to $(v, w)$, we obtain

$$
\left(\begin{array}{l}
\frac{\delta}{\delta v}\left(\frac{\partial \mathcal{S}}{\partial t}\right) \\
\frac{\delta}{\delta w}\left(\frac{\partial \mathcal{S}}{\partial t}\right)
\end{array}\right)=\left(\begin{array}{ll}
\left(\frac{\delta \vec{H}}{\delta v}\right)^{\top} & \left(\frac{\delta \vec{E}}{\delta v}\right)^{\top} \\
\left(\frac{\delta \vec{H}}{\delta w}\right)^{\top} & \left(\frac{\delta \vec{E}}{\delta w}\right)^{\top}
\end{array}\right)\left(\begin{array}{l}
\frac{\delta \mathcal{B}}{\delta \vec{H}} \\
\frac{\delta \mathcal{B}}{\delta \vec{E}}
\end{array}\right) .
$$

Differentiating the relation (49) with respect to $t$ yields

$$
\left(\begin{array}{c}
\frac{\partial}{\partial t}\left(\frac{\delta \mathcal{S}}{\delta v}\right) \\
\frac{\partial}{\partial t}\left(\frac{\delta \mathcal{S}}{\delta w}\right)
\end{array}\right)=\left(\begin{array}{cc}
I+\frac{1}{2} \frac{\delta^{2} \mathcal{S}}{\delta w \delta v} & -\frac{1}{2} \frac{\delta^{2} \mathcal{S}}{\delta v^{2}} \\
\frac{1}{2} \frac{\delta^{2} \mathcal{S}}{\delta w^{2}} & I-\frac{1}{2} \frac{\delta^{2} \mathcal{S}}{\delta v \delta w}
\end{array}\right)\left(\begin{array}{c}
-\frac{\partial \vec{E}}{\partial t}-\frac{\sigma_{1}+\sigma_{2}}{2} \vec{E} \\
\frac{\partial \vec{H}}{\partial t}+\frac{\sigma_{1}+\sigma_{2}}{2} \vec{H}
\end{array}\right) e^{\frac{\sigma_{1}+\sigma_{2}}{2} t}
$$

By comparing (55) with (56), and using (54), we get the Maxwell's equations in infinite dimensional Birkhoffian formulation (42). 
We assume that the Hamilton-Jacobi equation (51) has a solution of series:

$$
\mathcal{S}(v, w, t)=t \mathcal{G}_{1}(v, w, t)+t^{2} \mathcal{G}_{2}(v, w, t)+\cdots
$$

where $\mathcal{G}_{i}$ are functionals. Substituting (57) into (51) and comparing like powers of $t$ on the left and right sides of (51), we obtain a second order truncated series of $\mathcal{S}$ with $\mathcal{G}_{2}(v, w, t)=0$,

$$
\mathcal{S}(v, w, t) \approx t \mathcal{G}_{1}(v, w, t)=t \int_{X}\left(\frac{\sigma_{1}-\sigma_{2}}{2} v \cdot w-\frac{1}{2 \varepsilon} v \cdot \nabla \times v-\frac{1}{2 \mu} w \cdot \nabla \times w\right) d X .
$$

Substituting (58) into (49) we obtain a semi-discrete scheme with second order accuracy in time, it reads

$$
\begin{aligned}
\vec{E}^{k}-e^{\frac{\left(\sigma_{1}+\sigma_{2}\right) \tau}{2}} \vec{E}^{k+1} & =\tau\left[\frac{\sigma_{1}-\sigma_{2}}{4}\left(e^{\frac{\left(\sigma_{1}+\sigma_{2}\right) \tau}{2}} \vec{E}^{k+1}+\vec{E}^{k}\right)-\frac{1}{2 \varepsilon} \nabla \times\left(e^{\frac{\left(\sigma_{1}+\sigma_{2}\right) \tau}{2}} \vec{H}^{k+1}+\vec{H}^{k}\right)\right], \\
e^{\frac{\left(\sigma_{1}+\sigma_{2}\right) \tau}{2}} \vec{H}^{k+1}-\vec{H}^{k} & =\tau\left[\frac{\sigma_{1}-\sigma_{2}}{4}\left(e^{\frac{\left(\sigma_{1}+\sigma_{2}\right) \tau}{2}} \vec{H}^{k+1}+\vec{H}^{k}\right)-\frac{1}{2 \mu} \nabla \times\left(e^{\frac{\left(\sigma_{1}+\sigma_{2}\right) \tau}{2}} \vec{E}^{k+1}+\vec{E}^{k}\right)\right],
\end{aligned}
$$

where $\tau$ denotes the temporal step-size.

Theorem 3.1.2. If $(\vec{H}, \vec{E})$ satisfies periodic boundary condition or the boundary conditions (9), the semi-discrete scheme defined by (59) is of second order in temporal direction and is Birkhoffian symplectic scheme, i.e. it preserves the infinite dimensional 2-form (44) exactly.

Proof. Calculating exterior derivative of (59), we obtain

$$
\begin{aligned}
\delta \vec{E}^{k}-e^{\frac{\left(\sigma_{1}+\sigma_{2}\right) \tau}{2}} \delta \vec{E}^{k+1} & =\tau\left[\frac{\sigma_{1}-\sigma_{2}}{4}\left(e^{\frac{\left(\sigma_{1}+\sigma_{2}\right) \tau}{2}} \delta \vec{E}^{k+1}+\delta \vec{E}^{k}\right)-\frac{1}{2 \varepsilon} \nabla \times\left(e^{\frac{\left(\sigma_{1}+\sigma_{2}\right) \tau}{2}} \delta \vec{H}^{k+1}+\delta \vec{H}^{k}\right)\right], \\
e^{\frac{\left(\sigma_{1}+\sigma_{2}\right) \tau}{2}} \delta \vec{H}^{k+1}-\delta \vec{H}^{k} & =\tau\left[\frac{\sigma_{1}-\sigma_{2}}{4}\left(e^{\frac{\left(\sigma_{1}+\sigma_{2}\right) \tau}{2}} \delta \vec{H}^{k+1}+\delta \vec{H}^{k}\right)-\frac{1}{2 \mu} \nabla \times\left(e^{\frac{\left(\sigma_{1}+\sigma_{2}\right) \tau}{2}} \delta \vec{E}^{k+1}+\delta \vec{E}^{k}\right)\right] .
\end{aligned}
$$

using the boundary conditions, we have

$$
\begin{aligned}
& \int_{X} \nabla \times\left(e^{\frac{\left(\sigma_{1}+\sigma_{2}\right) \tau}{2}} \delta \vec{H}^{k+1}+\delta \vec{H}^{k}\right) \wedge\left(e^{\frac{\left(\sigma_{1}+\sigma_{2}\right) \tau}{2}} \delta \vec{H}^{k+1}+\delta \vec{H}^{k}\right) \\
= & \int_{X}\left(e^{\frac{\left(\sigma_{1}+\sigma_{2}\right) \tau}{2}} \delta \vec{H}^{k+1}+\delta \vec{H}^{k}\right) \wedge \nabla \times\left(e^{\frac{\left(\sigma_{1}+\sigma_{2}\right) \tau}{2}} \delta \vec{H}^{k+1}+\delta \vec{H}^{k}\right),
\end{aligned}
$$

and

$$
\begin{aligned}
& \int_{X} \nabla \times\left(e^{\frac{\left(\sigma_{1}+\sigma_{2}\right) \tau}{2}} \delta \vec{E}^{k+1}+\delta \vec{E}^{k}\right) \wedge\left(e^{\frac{\left(\sigma_{1}+\sigma_{2}\right) \tau}{2}} \delta \vec{E}^{k+1}+\delta \vec{E}^{k}\right) \\
= & \int_{X}\left(e^{\frac{\left(\sigma_{1}+\sigma_{2}\right) \tau}{2}} \delta \vec{E}^{k+1}+\delta \vec{E}^{k}\right) \wedge \nabla \times\left(e^{\frac{\left(\sigma_{1}+\sigma_{2}\right) \tau}{2}} \delta \vec{E}^{k+1}+\delta \vec{E}^{k}\right),
\end{aligned}
$$


which imply that (61) and (62) are zeros since $\wedge$ is skew-symmetric product. Left-wedging $\left(e^{\frac{\left(\sigma_{1}+\sigma_{2}\right) \tau}{2}} \delta \vec{H}^{k+1}+\delta \vec{H}^{k}, e^{\frac{\left(\sigma_{1}+\sigma_{2}\right) \tau}{2}} \delta \vec{E}^{k+1}+\delta \vec{E}^{k}\right)$ to $(60)$, we get

$$
\int_{X} e^{\left(\sigma_{1}+\sigma_{2}\right) \tau} \delta \vec{H}^{k+1} \wedge \delta \vec{E}^{k+1}=\int_{X} \delta \vec{H}^{k} \wedge \delta \vec{E}^{k}
$$

Suppose the solutions of the scheme (59) and the Maxwell's equations have the same form as following expansion

$$
\begin{aligned}
\vec{H}^{k+1} & =\vec{H}^{k}+\tau f_{1}\left(\vec{H}^{k}, \vec{E}^{k}\right)+\tau^{2} f_{2}\left(\vec{H}^{k}, \vec{E}^{k}\right)+\tau^{2} f_{3}\left(\vec{H}^{k}, \vec{E}^{k}\right)+\cdots, \\
\vec{E}^{k+1} & =\vec{E}^{k}+\tau g_{1}\left(\vec{H}^{k}, \vec{E}^{k}\right)+\tau^{2} g_{2}\left(\vec{H}^{k}, \vec{E}^{k}\right)+\tau^{2} g_{3}\left(\vec{H}^{k}, \vec{E}^{k}\right)+\cdots,
\end{aligned}
$$

then the solution of scheme (59) has the following coefficients

$$
\begin{aligned}
& f_{1}=-\sigma_{2} \vec{H}^{k}-\frac{1}{\mu} \nabla \times \vec{E}^{k}, \\
& f_{2}=\frac{\sigma_{2}^{2}}{2} \vec{H}^{k}+\frac{\sigma_{1}+\sigma_{2}}{2 \mu} \nabla \times \vec{E}^{k}-\frac{1}{2 \varepsilon \mu} \nabla \times\left(\nabla \times \vec{H}^{k}\right), \\
& f_{3}=a_{1} \vec{H}^{k}+b_{1} \nabla \times \vec{E}^{k}+c_{1} \nabla \times\left(\nabla \times \vec{H}^{k}\right)+\frac{1}{4 \varepsilon \mu^{2}} \nabla \times\left(\nabla \times\left(\nabla \times \vec{E}^{k}\right)\right),
\end{aligned}
$$

where

$$
a_{1}=\frac{\sigma_{1}^{3}-3 \sigma_{1}^{2} \sigma_{2}+3 \sigma_{1} \sigma_{2}^{2}-17 \sigma_{2}^{3}}{96}, \quad b_{1}=-\frac{3\left(\sigma_{1}+\sigma_{2}\right)^{2}-4 \sigma_{1} \sigma_{2}}{16 \mu}, \quad c_{1}=\frac{3 \sigma_{2}+\sigma_{1}}{8 \varepsilon \mu}
$$

and

$$
\begin{aligned}
& g_{1}=-\sigma_{1} \vec{E}^{k}+\frac{1}{\varepsilon} \nabla \times \vec{H}^{k}, \\
& g_{2}=\frac{\sigma_{1}^{2}}{2} \vec{E}^{k}-\frac{\sigma_{1}+\sigma_{2}}{2 \varepsilon} \nabla \times \vec{H}^{k}-\frac{1}{2 \varepsilon \mu} \nabla \times\left(\nabla \times \vec{E}^{k}\right), \\
& g_{3}=a_{2} \vec{E}^{k}+b_{2} \nabla \times \vec{H}^{k}+c_{2} \nabla \times\left(\nabla \times \vec{E}^{k}\right)-\frac{1}{4 \varepsilon^{2} \mu} \nabla \times\left(\nabla \times\left(\nabla \times \vec{H}^{k}\right)\right),
\end{aligned}
$$

where

$$
a_{2}=\frac{\sigma_{2}^{3}-3 \sigma_{2}^{2} \sigma_{1}+3 \sigma_{2} \sigma_{1}^{2}-17 \sigma_{1}^{3}}{96}, \quad b_{2}=\frac{3\left(\sigma_{1}+\sigma_{2}\right)^{2}-4 \sigma_{1} \sigma_{2}}{16 \varepsilon}, \quad c_{2}=\frac{3 \sigma_{1}+\sigma_{2}}{8 \varepsilon \mu} .
$$

And the exact solution of Maxwell's equations has different $f_{3}$ and $g_{3}$ as

$$
\begin{aligned}
& f_{3}=-\frac{\sigma_{2}^{3}}{6} \vec{H}^{k}-\frac{\sigma_{1}^{2}+\sigma_{2}^{2}+\sigma_{1} \sigma_{2}}{6 \mu} \nabla \times \vec{E}^{k}+\frac{\sigma_{1}+2 \sigma_{2}}{6 \varepsilon \mu} \nabla \times\left(\nabla \times \vec{H}^{k}\right)+\frac{1}{6 \varepsilon \mu^{2}} \nabla \times\left(\nabla \times\left(\nabla \times \vec{E}^{k}\right)\right), \\
& g_{3}=-\frac{\sigma_{1}^{3}}{6} \vec{E}^{k}+\frac{\sigma_{1}^{2}+\sigma_{2}^{2}+\sigma_{1} \sigma_{2}}{6 \varepsilon} \nabla \times \vec{H}^{k}+\frac{2 \sigma_{1}+\sigma_{2}}{6 \varepsilon \mu} \nabla \times\left(\nabla \times \vec{E}^{k}\right)-\frac{1}{6 \varepsilon^{2} \mu} \nabla \times\left(\nabla \times\left(\nabla \times \vec{H}^{k}\right)\right) .
\end{aligned}
$$

It shows that the scheme (59) is second order of accuracy in temporal direction. 
For convenience, we introduce some finite-difference operators as

$$
\begin{array}{rr}
R_{t} Z_{i, j, l}^{k}=\frac{1}{2}\left(Z_{i, j, l}^{k}+Z_{i, j, l}^{k+1}\right), & R_{1} Z_{i, j l}^{k}=\frac{1}{2}\left(Z_{i, j, l}^{k}+Z_{i+1, j, l}^{k}\right), \\
R_{2} Z_{i, j, l}^{k}=\frac{1}{2}\left(Z_{i, j, l}^{k}+Z_{i, j+1, l}^{k}\right), & R_{3} Z_{i, j, l}^{k}=\frac{1}{2}\left(Z_{i, j, l}^{k}+Z_{i, j, l+1}^{k}\right), \\
\partial_{t} Z_{i, j, l}^{k}=\frac{1}{\tau}\left(Z_{i, j, l}^{k+1}-Z_{i, j, l}^{k}\right), & \partial_{x} Z_{i, j, l}^{k}=\frac{1}{h_{x}}\left(Z_{i+1, j, l}^{k}-Z_{i, j l}^{k}\right), \\
\partial_{y} Z_{i, j, l}^{k}=\frac{1}{h_{y}}\left(Z_{i, j+1, l}^{k}-Z_{i, j, l}^{k}\right), & \partial_{z} Z_{i, j, l}^{k}=\frac{1}{h_{z}}\left(Z_{i, j, l+1}^{k}-Z_{i, j l l}^{k}\right), \\
\bar{\partial}_{t} Z_{i, j, l}^{k}=\frac{e^{\frac{\sigma_{1}+\sigma_{2}}{2} \tau} Z_{i, j, l}^{k+1}-Z_{i, j, l}^{k}}{\tau}, & \bar{R}_{t} Z_{i, j, l}^{k}=\frac{e^{\frac{\sigma_{1}+\sigma_{2}}{2} \tau} Z_{i, j, l}^{k+1}+Z_{i, j, l}^{k}}{2},
\end{array}
$$

where indices $(i, j, l)$ denote spatial increments and $k$ denotes temporal increment, $\left(h_{x}, h_{y}, h_{z}\right)$ denote spatial step-sizes. We denote the discrete curl operator at $(\mathrm{i}, \mathrm{j}, \mathrm{l}, \mathrm{k})$ to be

$$
\nabla \times Z_{i, j, l}^{k}=\left(A_{1} R_{2} R_{3} \partial_{x}+A_{2} R_{1} R_{3} \partial_{y}+A_{3} R_{1} R_{2} \partial_{z}\right) Z_{i, j, l}^{k} .
$$

All the operators have a commutative relationship. Applying midpoint rule in spatial direction to the semi-discrete system (59), we propose the Birkhoffian scheme as following

$$
\begin{array}{r}
-\bar{\partial}_{t} R_{1} R_{2} R_{3} \vec{E}_{i, j, l}^{k}+\frac{1}{\varepsilon}\left(A_{1} \partial_{x} \bar{R}_{t} R_{2} R_{3}+A_{2} \partial_{y} \bar{R}_{t} R_{1} R_{3}+A_{3} \partial_{z} \bar{R}_{t} R_{1} R_{2}\right) \vec{H}_{i, j, l}^{k} \\
=\frac{\sigma_{1}-\sigma_{2}}{2} \bar{R}_{t} R_{1} R_{2} R_{3} \vec{E}_{i, j, l}^{k}, \\
\bar{\partial}_{t} R_{1} R_{2} R_{3} \vec{H}_{i, j, l}^{k}+\frac{1}{\mu}\left(A_{1} \partial_{x} \bar{R}_{t} R_{2} R_{3}+A_{2} \partial_{y} \bar{R}_{t} R_{1} R_{3}+A_{3} \partial_{z} \bar{R}_{t} R_{1} R_{2}\right) \vec{E}_{i, j, l}^{k} \\
=\frac{\sigma_{1}-\sigma_{2}}{2} \bar{R}_{t} R_{1} R_{2} R_{3} \vec{H}_{i, j, l}^{k},
\end{array}
$$

Theorem 3.1.3. The Birkhoffian scheme (66) is Birkhoffian symplectic scheme as well as Birkhoffian multi-symplectic scheme.

Proof. The theorem 3.1.2 proves that the semi-discrete scheme (59) is symplectic, therefore the scheme (66) deduced by (59) is symplectic. By the definition 3.0.1, it is easy to prove the multi-symplecticity of the scheme (66), we omit it here.

\subsection{Central box scheme in autonomous Birkhoffian formulation}

Applying the central box scheme, which is also known as Preissman scheme, to the autonomous Birkhoffian system (21), we propose the Birkhoffian box scheme as following

$$
\begin{gathered}
-\partial_{t} R_{1} R_{2} R_{3} E_{i, j, l}^{k}+\frac{1}{\varepsilon_{1}\left(t^{k+\frac{1}{2}}\right)}\left(A_{1} \partial_{x} R_{t} R_{2} R_{3}+A_{2} \partial_{y} R_{t} R_{1} R_{3}+A_{3} \partial_{z} R_{t} R_{1} R_{2}\right) H_{i, j, l}^{k}=0, \\
\partial_{t} R_{1} R_{2} R_{3} H_{i, j, l}^{k}+\frac{1}{\mu_{1}\left(t^{k+\frac{1}{2}}\right)}\left(A_{1} \partial_{x} R_{t} R_{2} R_{3}+A_{2} \partial_{y} R_{t} R_{1} R_{3}+A_{3} \partial_{z} R_{t} R_{1} R_{2}\right) E_{i, j, l}^{k}=0 .
\end{gathered}
$$


It is obvious that there exists the following conclusion for the relationship between the Birkhoffian scheme (66) and the Birkhoffian box scheme (67).

Proposition 3.2.1. If $\sigma_{1}=\sigma_{2}$, the Birkhoffian scheme (66) can be transformed into the central box scheme (67) by the discrete version of the transformation (20).

Additionally, from the proposition 4.0.1 which will be introduced in section 4, we have the following theorem.

Theorem 3.2.1. The Birkhoffian box scheme (67) is Birkhoffian multi-symplectic scheme.

\subsection{Central box scheme in Hamiltonian formulation}

Applying the mid-point method in time direction to the Maxwell's equations with the Hamiltonian formulation (33), we get a semi-discrete system as

$$
\begin{aligned}
E^{k}-E^{k+1} & =\tau\left[\frac{\sigma_{1}-\sigma_{2}}{4}\left(E^{k+1}+E^{k}\right)-\frac{1}{2 \varepsilon} \nabla \times\left(H^{k+1}+H^{k}\right)\right], \\
H^{k+1}-H^{k} & =\tau\left[\frac{\sigma_{1}-\sigma_{2}}{4}\left(H^{k+1}+H^{k}\right)-\frac{1}{2 \mu} \nabla \times\left(E^{k+1}+E^{k}\right)\right] .
\end{aligned}
$$

The discrete version of transformation (32) is as

$$
\vec{H}^{k}=e^{-\frac{\sigma_{1}+\sigma_{2}}{2} t_{k}} H^{k}, \quad \vec{E}^{k}=e^{-\frac{\sigma_{1}+\sigma_{2}}{2} t_{k}} E^{k},
$$

by which the system (68) is transformed into the semi-discrete equations (59).

Applying the mid-point method in space direction to (68), we get the Hamiltonian box scheme:

$$
\begin{array}{r}
-\partial_{t} R_{1} R_{2} R_{3} E_{i, j, l}^{k}+\frac{1}{\varepsilon}\left(A_{1} \partial_{x} R_{t} R_{2} R_{3}+A_{2} \partial_{y} R_{t} R_{1} R_{3}+A_{3} \partial_{z} R_{t} R_{1} R_{2}\right) H_{i, j, l}^{k} \\
=\frac{\sigma_{1}-\sigma_{2}}{2} R_{t} R_{1} R_{2} R_{3} E_{i, j, l}^{k} \\
\partial_{t} R_{1} R_{2} R_{3} H_{i, j, l}^{k}+\frac{1}{\mu}\left(A_{1} \partial_{x} R_{t} R_{2} R_{3}+A_{2} \partial_{y} R_{t} R_{1} R_{3}+A_{3} \partial_{z} R_{t} R_{1} R_{2}\right) E_{i, j, l}^{k} \\
=\frac{\sigma_{1}-\sigma_{2}}{2} R_{t} R_{1} R_{2} R_{3} H_{i, j, l}^{k}
\end{array}
$$

The above scheme also can be derived by applying the central box scheme directly to the Hamiltonian formulation (33).

Proposition 3.3.1. The scheme (70) can be transformed into the Birkhoffian scheme (66) by the discrete transformation (69). 
Remark 3.3.1. The equivalence between (68) and (59) reveals the connection between the generating functional method (59) and the mid-point method which can be derived by the third type generating function. For more details of generating function method please refer to [26, 27, 28]. The generating functional method is a new developing theory proposed recently and needs more deep discussions.

\subsection{Central box method and exponential FDTD method in original form}

Applying the mid-point method in both temporal and spatial direction to the Maxwell's equations (1), we get the Central box scheme

$$
\begin{aligned}
-\partial_{t} R_{1} R_{2} R_{3} \vec{E}_{i, j, l}^{k}+\frac{1}{\varepsilon}\left(A_{1} \partial_{x} R_{t} R_{2} R_{3}+A_{2} \partial_{y} R_{t} R_{1} R_{3}\right. & \left.+A_{3} \partial_{z} R_{t} R_{1} R_{2}\right) \vec{H}_{i, j, l}^{k} \\
& =\sigma_{1} R_{t} R_{1} R_{2} R_{3} \vec{E}_{i, j, l}^{k} \\
\partial_{t} R_{1} R_{2} R_{3} \vec{H}_{i, j, l}^{k}+\frac{1}{\mu}\left(A_{1} \partial_{x} R_{t} R_{2} R_{3}+A_{2} \partial_{y} R_{t} R_{1} R_{3}\right. & \left.+A_{3} \partial_{z} R_{t} R_{1} R_{2}\right) \vec{E}_{i, j, l}^{k} \\
= & -\sigma_{2} R_{t} R_{1} R_{2} R_{3} \vec{H}_{i, j, l}^{k}
\end{aligned}
$$

Since the Maxwell's equations in original form (1) are neither Birkhoffian nor Hamiltonian, the scheme (71) does not preserve any (multi-)symplectic structure established above. Applying the exponential Yee's method (cf. [1]) to (1), we get the Exponential FDTD scheme,

$$
\begin{aligned}
\vec{E}^{k+1} & =e^{-\sigma_{1} \tau} \vec{E}^{k}+\frac{1-e^{-\sigma_{1} \tau}}{\varepsilon \sigma_{1}} \nabla \times \vec{H}^{k+\frac{1}{2}}, \\
\vec{H}^{k+\frac{1}{2}} & =e^{-\sigma_{2} \tau} \vec{H}^{k-\frac{1}{2}}+\frac{e^{-\sigma_{2} \tau}-1}{\mu \sigma_{2}} \nabla \times \vec{E}^{k}
\end{aligned}
$$

where we have the same space nodes and discrete curl operator as that in traditional Yee's method.

\section{Energy-conservation, stability, dissipation and dispersion analysis}

In this section, we discuss energy-preserving property, stability, dissipation and dispersion relations of the Birkhoffian scheme (66) and the Birkhoffian box scheme (67). At first we give a conservation property of multi-symplectic partitioned Runge-Kutta (MSPRK) method. We consider an autonomous system of Birkhoffian PDEs

$$
M(x) u_{t}+K(t) u_{x}=\nabla S(u, x, t),
$$


applying a (r,s)-stage partitioned Runge-Kutta (PRK) method to (73), we have

$$
\begin{array}{r}
u_{m}^{1}=u_{m}^{0}+\tau \sum_{k=1}^{r} \bar{b}_{k} \partial_{t} U_{m}^{k}, \\
U_{m}^{k}=u_{m}^{0}+\tau \sum_{j=1}^{r} \bar{a}_{k j} \partial_{t} U_{m}^{j}, \\
u_{1}^{k}=u_{0}^{k}+h \sum_{m=1}^{s} b_{m} \partial_{x} U_{m}^{k}, \\
U_{m}^{k}=u_{0}^{k}+h \sum_{n=1}^{s} a_{m n} \partial_{x} U_{n}^{k}, \\
M\left(x_{m}\right) \partial_{t} U_{m}^{k}+K\left(t_{k}\right) \partial_{x} U_{m}^{k}=\nabla S\left(U_{m}^{k}, x_{m}, t_{k}\right),
\end{array}
$$

where $h$ is space step-size and $\tau$ is time step-size. Let $U_{m}^{k} \approx u\left(x_{m}, t_{k}\right), u_{m}^{1} \approx u\left(x_{m}, \tau\right)$, $u_{m}^{0} \approx u\left(x_{m}, 0\right)$, and $u_{1}^{k} \approx u\left(h, t_{k}\right), u_{0}^{k} \approx u\left(0, t_{k}\right) . c_{m}=\sum_{n=1}^{s} a_{m n}$ and $\bar{c}_{k}=\sum_{j=1}^{r} \bar{a}_{k j}$, $x_{m}=c_{m} h, t_{k}=\bar{c}_{k} \tau . \partial_{t} U_{m}^{k} \approx u_{t}\left(x_{m}, t_{k}\right)$ and $\partial_{x} U_{m}^{k} \approx u_{x}\left(x_{m}, t_{k}\right)$ are the discretizations of $\partial_{t}$ and $\partial_{x}$. We know that a PRK method is a MSPRK method for Hamiltonian PDEs if and only if the coefficients satisfy the following multi-symplectic conditions,

$$
\bar{b}_{k} \bar{b}_{j}-\bar{b}_{k} \bar{a}_{k j}-\bar{b}_{j} \bar{a}_{j k}=0 \text { and } b_{m} b_{n}-b_{m} a_{m n}-b_{n} a_{n m}=0
$$

Proposition 4.0.1. If the coefficients in the numerical scheme (74) satisfy the multisymplectic conditions (75) for $k, j=1,2, \cdots, r$ and $m, n=1,2, \cdots, s$, then (74) preserves the discrete multi-symplectic conservation law

$$
\begin{aligned}
h \sum_{m=1}^{s} b_{m}\left(d u_{m}^{1} \wedge M\left(x_{m}\right) d u_{m}^{1}-d u_{m}^{0} \wedge M\left(x_{m}\right) d u_{m}^{0}\right) & + \\
\tau \sum_{k=1}^{r} \bar{b}_{k}\left(d u_{1}^{k} \wedge K\left(t_{k}\right) d u_{1}^{k}-d u_{0}^{k} \wedge K\left(t_{k}\right) d u_{0}^{k}\right) & =0 .
\end{aligned}
$$

Since the discrete versions $M\left(x_{m}\right)$ is independent of time grid index $k$ and $K\left(t_{k}\right)$ independent of space grid index $m$, the proof of this theorem is similar to the proof of multisymplecticity of MSPRK method applied to Hamiltonian PDEs, therefore omitted here.

In what follows, we give an important result for MSPRK method implemented to a special case of Hamiltonian PDEs as following

$$
M u_{t}+K u_{x}=0
$$


We assume there exists a vector function $\phi\left(u, u_{x}, u_{t}, u_{x t}\right)$ such that

$$
\phi\left(u, u_{x}, u_{t}, u_{x t}\right)^{\top}\left(M u_{t}+K u_{x}\right)=\partial_{t} \xi(u)+\partial_{x} \eta(u)
$$

where $\xi(u)=u_{x}^{\top} L_{1} u_{x}, \eta(u)=u_{t}^{\top} L_{2} u_{t}$ and $L_{1}$ and $L_{2}$ are symmetric matrices, then

$$
\partial_{t} \xi(u)+\partial_{x} \eta(u)=0
$$

is a quadratic conservation law of (77).

Example 4.0.1. If the matrix $K$ is invertible, we let $\phi=K^{-\top} u_{x t}$, then

$$
\phi^{\top} \cdot\left(M u_{t}+K u_{x}\right)=u_{x t}^{\top} K^{-1} M u_{t}+u_{x t}^{\top} u_{x}
$$

since $M$ and $K$ are skew-symmetric matrices, $K^{-1} M$ is a symmetric matrix. Then we have a conservation law $\partial_{t} \xi(u)+\partial_{x} \eta(u)=0$ with $\xi(u)=\frac{1}{2} u_{x}^{\top} u_{x}$ and $\eta(u)=\frac{1}{2} u_{t}^{\top} K^{-1} M u_{t}$.

Proposition 4.0.2. Applying a $(r, s)$-stage $M S P R K$ method to $(77)$, if $A=\left(a_{m n}\right)_{s \times s}$ and $\bar{A}=\left(\bar{a}_{k j}\right)_{r \times r}$ are invertible, the resulted numerical scheme preserves the discrete version of the conservation law (79) as following

$$
h \sum_{m=1}^{s} b_{m}\left(\xi\left(u_{m}^{1}\right)-\xi\left(u_{m}^{0}\right)\right)+\tau \sum_{k=1}^{r} \bar{b}_{k}\left(\eta\left(u_{1}^{k}\right)-\eta\left(u_{0}^{k}\right)\right)=0,
$$

with

$$
\begin{array}{cl}
\xi\left(u_{m}^{1}\right)=\left(\left(\partial_{x} u\right)_{m}^{1}\right)^{\top} L_{1}\left(\partial_{x} u\right)_{m}^{1}, & \xi\left(u_{m}^{0}\right)=\left(\left(\partial_{x} u\right)_{m}^{0}\right)^{\top} L_{1}\left(\partial_{x} u\right)_{m}^{0}, \\
\eta\left(u_{1}^{k}\right)=\left(\left(\partial_{t} u\right)_{1}^{k}\right)^{\top} L_{2}\left(\partial_{t} u\right)_{1}^{k}, & \eta\left(u_{0}^{k}\right)=\left(\left(\partial_{t} u\right)_{0}^{k}\right)^{\top} L_{2}\left(\partial_{t} u\right)_{0}^{k},
\end{array}
$$

where $\left(\partial_{t} u\right)_{m}^{1},\left(\partial_{t} u\right)_{m}^{0},\left(\partial_{x} u\right)_{1}^{k}$ and $\left(\partial_{x} u\right)_{0}^{k}$ satisfy the following system

$$
\begin{aligned}
&\left(\partial_{x} u\right)_{m}^{1}=\left(\partial_{x} u\right)_{m}^{0}+\tau \sum_{k=1}^{r} \bar{b}_{k} \partial_{t}\left(\partial_{x} U_{m}^{k}\right), \\
& \partial_{x} U_{m}^{k}=\left(\partial_{x} u\right)_{m}^{0}+\tau \sum_{j=1}^{r} \bar{a}_{k j} \partial_{t}\left(\partial_{x} U_{m}^{j}\right), \\
&\left(\partial_{t} u\right)_{1}^{k}=\left(\partial_{t} u\right)_{0}^{k}+h \sum_{m=1}^{s} b_{m} \partial_{x}\left(\partial_{t} U_{m}^{k}\right), \\
& \partial_{t} U_{m}^{k}=\left(\partial_{t} u\right)_{0}^{k}+h \sum_{n=1}^{s} a_{m n} \partial_{x}\left(\partial_{t} U_{n}^{k}\right),
\end{aligned}
$$


and

$$
u_{m}^{0}=u_{0}^{0}+h \sum_{n=1}^{s} a_{m n}\left(\partial_{x} u\right)_{n}^{0}, \quad u_{0}^{k}=u_{0}^{0}+\tau \sum_{j=1}^{r} \bar{a}_{k j}\left(\partial_{t} u\right)_{0}^{j} .
$$

Here we use notations $\partial_{t}\left(\partial_{x} U_{m}^{k}\right) \approx \partial_{x t} u\left(x_{m}, t_{k}\right)$ and $\partial_{x}\left(\partial_{t} U_{m}^{k}\right) \approx \partial_{t x} u\left(x_{m}, t_{k}\right)$.

Proof. From (74), (83) and (84) we have

$$
\begin{aligned}
U_{m}^{k} & =u_{m}^{0}+\tau \sum_{j=1}^{r} \bar{a}_{k j}\left[\left(\partial_{t} u\right)_{0}^{j}+h \sum_{n=1}^{s} a_{m n} \partial_{x}\left(\partial_{t} U_{n}^{j}\right)\right] \\
& =u_{m}^{0}+u_{0}^{k}-u_{0}^{0}+h \tau \sum_{j=1}^{r} \sum_{n=1}^{s} \bar{a}_{k j} a_{m n} \partial_{x}\left(\partial_{t} U_{n}^{j}\right),
\end{aligned}
$$

and

$$
\begin{aligned}
U_{m}^{k} & =u_{0}^{k}+h \sum_{n=1}^{s} a_{m n}\left[\left(\partial_{x} u\right)_{n}^{0}+\tau \sum_{j=1}^{r} \bar{a}_{k j} \partial_{t}\left(\partial_{x} U_{n}^{j}\right)\right] \\
& =u_{0}^{k}+u_{m}^{0}-u_{0}^{0}+h \tau \sum_{n=1}^{s} \sum_{j=1}^{r} a_{m n} \bar{a}_{k j} \partial_{t}\left(\partial_{x} U_{n}^{j}\right),
\end{aligned}
$$

by the invertibility of $A$ and $\bar{A}$, we have $\partial_{t}\left(\partial_{x} U_{n}^{j}\right)=\partial_{x}\left(\partial_{t} U_{n}^{j}\right)$ for $n=1,2, \cdots, s$ and $j=1,2, \cdots, r$. Using (83), we obtain via (82)

$$
\begin{aligned}
\xi\left(u_{m}^{1}\right)=\xi\left(u_{m}^{0}\right) & +\tau \sum_{k=1}^{r} \bar{b}_{k}\left(\left(\partial_{x} u\right)_{m}^{0}\right)^{\top} L_{1} \partial_{t}\left(\partial_{x} U_{m}^{k}\right) \\
& +\tau \sum_{k=1}^{r} \bar{b}_{k}\left(\partial_{t}\left(\partial_{x} U_{m}^{k}\right)\right)^{\top} L_{1}\left(\partial_{x} u\right)_{m}^{0} \\
& +\tau^{2} \sum_{k=1}^{r} \sum_{j=1}^{r} \bar{b}_{k} \bar{b}_{j}\left(\partial_{t}\left(\partial_{x} U_{m}^{k}\right)\right)^{\top} L_{1} \partial_{t}\left(\partial_{x} U_{m}^{j}\right)
\end{aligned}
$$

which reduces to the following expression by (83)

$$
\begin{aligned}
\xi\left(u_{m}^{1}\right)-\xi\left(u_{m}^{0}\right) & =2 \tau \sum_{k=1}^{r} \bar{b}_{k}\left(\partial_{x} U_{m}^{k}\right)^{\top} L_{1} \partial_{t}\left(\partial_{x} U_{m}^{k}\right) \\
& +\tau^{2} \sum_{k=1}^{r} \sum_{j=1}^{r}\left(\bar{b}_{k} \bar{b}_{j}-\bar{b}_{k} \bar{a}_{k j}-\bar{b}_{j} \bar{a}_{j k}\right)\left(\partial_{t}\left(\partial_{x} U_{m}^{k}\right)\right)^{\top} L_{1} \partial_{t}\left(\partial_{x} U_{m}^{j}\right),
\end{aligned}
$$

by the multi-symplectic conditions (75), this is simplified into

$$
\xi\left(u_{m}^{1}\right)-\xi\left(u_{m}^{0}\right)=2 \tau \sum_{k=1}^{r} \bar{b}_{k}\left(\partial_{x} U_{m}^{k}\right)^{\top} L_{1} \partial_{t}\left(\partial_{x} U_{m}^{k}\right),
$$


taking the similar calculation, we derive

$$
\eta\left(u_{1}^{k}\right)-\eta\left(u_{0}^{k}\right)=2 h \sum_{m=1}^{s} b_{m}\left(\partial_{t} U_{m}^{k}\right)^{\top} L_{2} \partial_{x}\left(\partial_{t} U_{m}^{k}\right) .
$$

Combining (89) and (90), we conclude

$$
\begin{aligned}
& h \sum_{m=1}^{s} b_{m}\left(\xi\left(u_{m}^{1}\right)-\xi\left(u_{m}^{0}\right)\right)+\tau \sum_{k=1}^{r} \bar{b}_{k}\left(\eta\left(u_{1}^{k}\right)-\eta\left(u_{0}^{k}\right)\right)= \\
& 2 h \tau \sum_{m=1}^{s} \sum_{k=1}^{r} b_{m} \bar{b}_{k}\left[\left(\partial_{x} U_{m}^{k}\right)^{\top} L_{1} \partial_{t}\left(\partial_{x} U_{m}^{k}\right)+\left(\partial_{t} U_{m}^{k}\right)^{\top} L_{2} \partial_{x}\left(\partial_{t} U_{m}^{k}\right)\right] .
\end{aligned}
$$

Using the relationship (78) and $\partial_{t}\left(\partial_{x} U_{n}^{j}\right)=\partial_{x}\left(\partial_{t} U_{n}^{j}\right)$, we have

$$
\begin{array}{r}
\phi^{\top}\left(U_{m}^{k}, \partial_{x} U_{m}^{k}, \partial_{t} U_{m}^{k}, \partial_{t}\left(\partial_{x} U_{m}^{k}\right)\right)\left(M \partial_{t} U_{m}^{k}+\partial_{x} U_{m}^{k}\right)= \\
\left(\partial_{x} U_{m}^{k}\right)^{\top} L_{1} \partial_{t}\left(\partial_{x} U_{m}^{k}\right)+\left(\partial_{t} U_{m}^{k}\right)^{\top} L_{2} \partial_{x}\left(\partial_{t} U_{m}^{k}\right)
\end{array}
$$

From $M \partial_{t} U_{m}^{k}+K \partial_{x} U_{m}^{k}=0$, we conclude (81).

\subsection{Formal energy/momentum conservation}

The central box method applied to Hamiltonian PDEs is a (1,1)-stage MSPRK method. From the results in $[8,17,21]$ and the proposition 4.0 .2 , we obtain the following conclusions for the difference system (70):

Proposition 4.1.1. The scheme (70) preserves the discrete versions of multi-symplectic energy and momentum conservation laws (37) and (39). If $\sigma_{1}=\sigma_{2}$, it also preserves the discrete versions of the quadratic conservation law (27) and (28).

Proof. Since (37) and (39) are multi-symplectic energy and momentum conservation laws for the Hamiltonian PDEs, the proposition holds for (37) and (39) by generalization to high spatial dimensions the propositions in [17] and the theorem in [21]. (27) is a quadratic local conservation law, according to the result in cf. [8], a MSPRK method applied to Hamiltonian PDEs is quadratic invariant-preserving, therefore the proposition holds for (27) from the result in cf. [8]. From the result of proposition 4.0.2, the conservation law (28) can be also conserved by the scheme (70).

By the proposition 3.3.1, the conservation laws (8), (14), (17) and (19) can be transformed respectively into (27), (28), (37) and (39). Therefore, using the result in Proposition 4.1.1, we come up the following conclusions. 
Corollary 4.1.1. The Birkhoffian scheme (66) preserves exactly the multi-symplectic energy and momentum conservation laws (17) and (19). When $\sigma_{1}=\sigma_{2}$, it also preserves exactly the quadratic conservation laws (8) and (14).

Corollary 4.1.2. If $\sigma_{1}=\sigma_{2}$, the Birkhoffian box scheme (67) preserves exactly the local conservation laws (25)-(28).

Here we give the discrete version of the local conservation laws (25), (27) and (28). The discrete version of $(25)$ is

$$
\begin{array}{r}
\partial_{t}\left(\frac{1}{\varepsilon} R_{1} R_{2} R_{3} H_{i, j, l}^{k} \cdot \nabla \times H_{i, j, l}^{k}+\frac{1}{\mu} R_{1} R_{2} R_{3} E_{i, j, l}^{k} \cdot \nabla \times E_{i, j, l}^{k}\right) \\
+\partial_{x}\left(\frac{1}{\varepsilon} \partial_{t} R_{2} R_{3} H_{i, j, l}^{k} \cdot A_{1} R_{t} R_{2} R_{3} H_{i, j, l}^{k}+\frac{1}{\mu} \partial_{t} R_{2} R_{3} E_{i, j, l}^{k} \cdot A_{1} R_{t} R_{2} R_{3} E_{i, j, l}^{k}\right) \\
+\partial_{y}\left(\frac{1}{\varepsilon} \partial_{t} R_{1} R_{3} H_{i, j, l}^{k} \cdot A_{2} R_{t} R_{1} R_{3} H_{i, j l}^{k}+\frac{1}{\mu} \partial_{t} R_{1} R_{3} E_{i, j, l}^{k} \cdot A_{2} R_{t} R_{1} R_{3} E_{i, j, l}^{k}\right) \\
+\partial_{z}\left(\frac{1}{\varepsilon} \partial_{t} R_{1} R_{2} H_{i, j, l}^{k} \cdot A_{3} R_{t} R_{1} R_{2} H_{i, j, l}^{k}+\frac{1}{\mu} \partial_{t} R_{1} R_{2} E_{i, j, l}^{k} \cdot A_{3} R_{t} R_{1} R_{2} E_{i, j, l}^{k}\right)=0,
\end{array}
$$

the discrete version of $(27)$ is

$$
\begin{aligned}
0=\frac{1}{2} \partial_{t}\left(\varepsilon \mid R_{1}\right. & \left.\left.R_{2} R_{3} E_{i, j, l}^{k}\right|^{2}+\mu\left|R_{1} R_{2} R_{3} H i, j, l^{k}\right|^{2}\right) \\
& +\partial_{x}\left(R_{t} R_{2} R_{3} H_{i, j, l}^{k} \cdot A_{1} R_{t} R_{2} R_{3} E_{i, j, l}^{k}\right) \\
& +\partial_{y}\left(R_{t} R_{1} R_{3} H_{i, j, l}^{k} \cdot A_{2} R_{t} R_{1} R_{3} E_{i, j, l}^{k}\right) \\
& +\partial_{z}\left(R_{t} R_{1} R_{2} H_{i, j, l}^{k} \cdot A_{3} R_{t} R_{1} R_{2} E_{i, j, l}^{k}\right),
\end{aligned}
$$

and the discrete version of (28) reads

$$
\begin{aligned}
0=\partial_{t} & \left(\frac{1}{2 \varepsilon}\left|\nabla \times H_{i, j, l}^{k}\right|^{2}+\frac{1}{2 \mu}\left|\nabla \times E_{i, j, l}^{k}\right|^{2}\right) \\
& +\partial_{x}\left(\partial_{t} R_{2} R_{3} H_{i, j, l}^{k} \cdot A_{1} \partial_{t} R_{2} R_{3} E_{i, j, l}^{k}\right) \\
& +\partial_{y}\left(\partial_{t} R_{1} R_{3} H_{i, j, l}^{k} \cdot A_{2} \partial_{t} R_{1} R_{3} E_{i, j, l}^{k}\right) \\
& +\partial_{z}\left(\partial_{t} R_{1} R_{2} H_{i, j, l}^{k} \cdot A_{3} \partial_{t} R_{1} R_{2} E_{i, j, l}^{k}\right),
\end{aligned}
$$

where $H^{k}=e^{\frac{\sigma_{1}+\sigma_{2}}{2} \tau} \vec{H}^{k}$ and $E^{k}=e^{\frac{\sigma_{1}+\sigma_{2}}{2} \tau} \vec{E}^{k}$. Similarly, we can derive the discrete version of multisymplectic momentum conservation law (26).

From the discrete local conservation laws in the corollaries 4.1.1 and 4.1.2, we have the following conclusions for discrete global conservation laws. 
Theorem 4.1.1. The Birkhoffian scheme (66) with periodic boundary conditions preserves the global helicity conservation law as

$$
\begin{array}{r}
h_{x} h_{y} h_{z} \sum_{i, j, l} \bar{\partial}_{t}\left(\frac{1}{\varepsilon} R_{1} R_{2} R_{3} \vec{H}_{i, j, l}^{k} \cdot \nabla \times \vec{H}_{i, j, l}^{k}+\frac{1}{\mu} R_{1} R_{2} R_{3} \vec{E}_{i, j, l}^{k} \cdot \nabla \times \vec{E}_{i, j, l}^{k}\right. \\
\left.+\frac{\sigma_{2}-\sigma_{1}}{2} R_{1} R_{2} R_{3} \vec{H}_{i, j, l}^{k} \cdot R_{1} R_{2} R_{3} \vec{E}_{i, j, l}^{k}\right)=0 .
\end{array}
$$

When $\sigma_{1}=\sigma_{2}$, (66) also preserves the global quadratic conservation law (10) as

$$
h_{x} h_{y} h_{z} \sum_{i, j, l} \bar{\partial}_{t}\left(\mu\left|R_{1} R_{2} R_{3} \vec{H}_{i, j, l}^{k}\right|^{2}+\varepsilon\left|R_{1} R_{2} R_{3} \vec{E}_{i, j, l}^{k}\right|^{2}\right)=0,
$$

and preserves the discrete version of (15) as

$$
h_{x} h_{y} h_{z} \sum_{i, j, l} \bar{\partial}_{t}\left(\frac{1}{\varepsilon}\left|\nabla \times \vec{H}_{i, j, l}^{k}\right|^{2}+\frac{1}{\mu}\left|\nabla \times \vec{E}_{i, j, l}^{k}\right|^{2}\right)=0 .
$$

Theorem 4.1.2. If $\sigma_{1}=\sigma_{2}$, the Birkhoffian box scheme (67) with periodic boundary condition preserves the following three discretized conservation laws:

$$
\begin{gathered}
h_{x} h_{y} h_{z} \sum_{i, j, l} \partial_{t}\left(\frac{1}{\varepsilon} R_{1} R_{2} R_{3} H_{i, j, l}^{k} \cdot \nabla \times H_{i, j l}^{k}+\frac{1}{\mu} R_{1} R_{2} R_{3} E_{i, j, l}^{k} \cdot \nabla \times E_{i, j, l}^{k}\right)=0, \\
h_{x} h_{y} h_{z} \sum_{i, j, l} \partial_{t}\left(\varepsilon\left|R_{1} R_{2} R_{3} E_{i, j, l}^{k}\right|^{2}+\mu\left|R_{1} R_{2} R_{3} H_{i, j, l}^{k}\right|^{2}\right)=0,
\end{gathered}
$$

and

$$
h_{x} h_{y} h_{z} \sum_{i, j, l} \partial_{t}\left(\frac{1}{2 \varepsilon}\left|\nabla \times H_{i, j, l}^{k}\right|^{2}+\frac{1}{2 \mu}\left|\nabla \times E_{i, j, l}^{k}\right|^{2}\right)=0 .
$$

\subsection{Stability and dissipation}

Suppose the Birkhoffian scheme (66) has a solution of the form

$$
\left(\begin{array}{c}
\vec{E}_{i j l}^{k} \\
\vec{H}_{i j l}^{k}
\end{array}\right)=\rho^{k} e^{-\sqrt{-1}\left(i \kappa_{x} h_{x}+j \kappa_{y} h_{y}+l \kappa_{z} h_{z}\right)}\left(\begin{array}{c}
\vec{E}_{0} \\
\vec{H}_{0}
\end{array}\right)
$$

where $(\vec{E}, \vec{H})$ is the eigenvector of the scheme (66), and $\rho$ is the stability factor depending on time whose modulus will determine the stability and dissipation of (66) under discussion, and $\left(\kappa_{x}, \kappa_{y}, \kappa_{z}\right)$ is the vector wave number. Substituting (100) into the scheme yields

$$
\left(\begin{array}{cc}
\zeta_{1} I & \eta_{1} S \\
\eta_{2} S & \zeta_{2} I
\end{array}\right)\left(\begin{array}{l}
\vec{E}_{0} \\
\vec{H}_{0}
\end{array}\right)=0
$$


where $I$ is identity matrix, $\zeta_{i}$ and $\eta_{i}(i=1,2)$ are coefficients,

$$
\begin{aligned}
& \zeta_{1}=\left[4+\left(\sigma_{1}-\sigma_{2}\right) \tau\right] \rho e^{\bar{\sigma} \tau}+\left(\sigma_{1}-\sigma_{2}\right) \tau-4, \quad \eta_{1}=\frac{1+\rho e^{\bar{\sigma} \tau}}{\varepsilon} \tau \sqrt{-1}, \\
& \left.\zeta_{2}=\left[-4+\left(\sigma_{1}-\sigma_{2}\right) \tau\right] \rho e^{\bar{\sigma} \tau}+\left(\sigma_{1}-\sigma_{2}\right) \tau+4\right], \quad \eta_{2}=\frac{1+\rho e^{\bar{\sigma} \tau}}{\mu} \tau \sqrt{-1}
\end{aligned}
$$

and

$$
S=\frac{s_{1}}{h_{x}} A_{1}+\frac{s_{2}}{h_{y}} A_{2}+\frac{s_{3}}{h_{z}} A_{3}
$$

where

$$
\begin{aligned}
& s_{1}=\left(\sin \theta_{1}-\sin \theta_{2}+\sin \theta_{3}+\sin \theta_{4}\right) /\left(\cos \theta_{1}+\cos \theta_{2}+\cos \theta_{3}+\cos \theta_{4}\right), \\
& s_{2}=\left(\sin \theta_{1}+\sin \theta_{2}+\sin \theta_{3}-\sin \theta_{4}\right) /\left(\cos \theta_{1}+\cos \theta_{2}+\cos \theta_{3}+\cos \theta_{4}\right), \\
& s_{3}=\left(\sin \theta_{1}+\sin \theta_{2}-\sin \theta_{3}+\sin \theta_{4}\right) /\left(\cos \theta_{1}+\cos \theta_{2}+\cos \theta_{3}+\cos \theta_{4}\right),
\end{aligned}
$$

with $\theta_{1}=\frac{x+y+z}{2}, \theta_{2}=\frac{-x+y+z}{2}, \theta_{3}=\frac{x+y-z}{2}, \theta_{4}=\frac{x-y+z}{2}$. Since the equality (101) holds for nonzero vector $\left(\vec{E}_{0}, \vec{H}_{0}\right)$, this implies that the coefficient determinant of (101) is zero, that is

$$
\zeta_{1}^{3}\left|\zeta_{2} I-\frac{\eta_{1} \eta_{2}}{\zeta_{1}} S^{2}\right|=0 .
$$

If $16 c^{2}-\varepsilon \mu\left(\sigma_{1}-\sigma_{2}\right)^{2} \geq 0$, we calculate the magnitude of the characteristic roots

$$
\begin{aligned}
& \left|\rho_{1}\right|=e^{-\bar{\sigma} \tau}, \\
& \left|\rho_{2}\right|=\left|\frac{4 \pm\left(\sigma_{1}-\sigma_{2}\right) \tau}{4 \mp\left(\sigma_{1}-\sigma_{2}\right) \tau}\right| e^{-\bar{\sigma} \tau},
\end{aligned}
$$

where $c^{2}=\frac{s_{1}^{2}}{h_{x}^{2}}+\frac{s_{2}^{2}}{h_{y}^{2}}+\frac{s_{3}^{2}}{h_{z}^{2}}$. If $16 c^{2}-\varepsilon \mu\left(\sigma_{1}-\sigma_{2}\right)^{2}<0$, we get

$$
\begin{aligned}
\left|\rho_{1}\right| & =e^{-\bar{\sigma} \tau}, \\
\left|\rho_{2}\right| & =\left|\frac{4 \pm\left(\sigma_{1}-\sigma_{2}\right) \tau}{4 \mp\left(\sigma_{1}-\sigma_{2}\right) \tau}\right| e^{-\bar{\sigma} \tau}, \\
\left|\rho_{3}\right| & =\left|\Phi \pm \sqrt{\Phi^{2}-1}\right| e^{-\bar{\sigma} \tau}, \\
\text { where } \Phi & =\frac{16 \varepsilon \mu-\left[16 c^{2}-\varepsilon \mu\left(\sigma_{1}-\sigma_{2}\right)^{2}\right] \tau^{2}}{16 \varepsilon \mu+\left[16 c^{2}-\varepsilon \mu\left(\sigma_{1}-\sigma_{2}\right)^{2}\right] \tau^{2}}
\end{aligned}
$$

It is easy to verify that $\left|\rho_{i}\right| \leq e^{-\bar{\sigma} \tau}$ holds in the case of $\left|\sigma_{1}-\sigma_{2}\right| \ll 1$, especially, $\left|\rho_{i}\right|=e^{-\bar{\sigma} \tau}$ if $\sigma_{1}=\sigma_{2}$. Combining with the above discussion, we know that the Birkhoffian scheme (66) is conditionally stable in the case of $\sigma_{1} \neq \sigma_{2}$ and unconditionally stable for $\sigma_{1}=\sigma_{2}$. We conclude the following theorem: 
Theorem 4.2.1. If $\sigma_{1}=\sigma_{2}=\bar{\sigma}$, the scheme (66) is unconditionally stable and $e^{-\bar{\sigma} \tau}$ dissipative; If $0<\left|\sigma_{1}-\sigma_{2}\right| \ll 1$, it is stable and dissipative.

We remark that $e^{-\bar{\sigma} \tau}$-dissipation implies that scheme (66) is dissipation-preserving.

Similarly, substituting a solution of the form

$$
\left(\begin{array}{c}
E_{i j l}^{k} \\
H_{i j l}^{k}
\end{array}\right)=\rho^{k} e^{-\sqrt{-1}\left(i \kappa_{x} h_{x}+j \kappa_{y} h_{y}+l \kappa_{z} h_{z}\right)}\left(\begin{array}{c}
E_{0} \\
H_{0}
\end{array}\right)
$$

into the scheme (67), we get

$$
\left(\begin{array}{cc}
(\rho-1) I & \frac{\sqrt{-1} \tau}{4 \varepsilon_{1}}(\rho+1) S \\
\frac{\sqrt{-1} \tau}{4 \mu_{1}}(\rho+1) S & (1-\rho) I
\end{array}\right)\left(\begin{array}{l}
\vec{E}_{0} \\
\vec{H}_{0}
\end{array}\right)=0
$$

the character roots can be derived as

$$
\begin{aligned}
\rho_{1} & =1 \\
\rho_{2}^{2}-2 \frac{\varepsilon_{1} \mu_{1}-c^{2} \tau^{2}}{\varepsilon_{1} \mu_{2}+c^{2} \tau^{2}} \rho_{2}+1 & =0
\end{aligned}
$$

it is easy to verify that $\rho_{2}$ has to be imaginary number and $\left|\rho_{2}\right|=1$.

Theorem 4.2.2. The scheme (67) is unconditionally stable and non-dissipative.

It is obviously that the non-dissipation property of the autonomous Bikhoffian system (21) is preserved by the scheme (67).

By repeating the same discussion, we conclude that scheme (71) is conditionally stable, and unconditionally stable only if $\sigma_{1}=\sigma_{2}$, while the central box scheme implemented to Maxwell's equations without dissipation terms is unconditionally stable.

Theorem 4.2.3. If $\sigma_{1}=\sigma_{2}$, the scheme (71) is unconditionally stable and non-dissipative.

The non-dissipative property of scheme (71) implies that the scheme connot preserve the dissipation property of the electromagnetic field.

\subsection{Dispersion relations}

There is a large amount of literature on the dispersion analysis of differential equations and numerical methods. For instance, see [29] for dispersion analysis of numerical methods for wave equations, and see [30] for dispersion analysis of FDTD method for Maxwell's equations. In this section, we follow the general dispersion analysis for difference equations 
in cf. [29] to discuss the discrete dispersion relations of the Birkhoffian scheme and the Birkhoffian box scheme.

Suppose the Maxwell's equations (1) has a solution of the following form:

$$
\vec{H}=e^{-\sigma_{2} t} \vec{H}_{0} e^{i(\omega t-\alpha x-\beta y-\gamma z)}, \quad \vec{E}=e^{-\sigma_{1} t} \vec{E}_{0} e^{i(\omega t-\alpha x-\beta y-\gamma z)},
$$

where $\vec{H}_{0}$ and $\vec{E}_{0}$ denote any constant vectors, $\omega, \alpha, \beta$ and $\gamma$ are real constants. $e^{-\sigma_{1} t}$ and $e^{-\sigma_{2} t}$ imply that $\vec{H}$ and $\vec{E}$ attenuate with time. Maxwell's equations (1) also has another form of solutions which attenuate with space. In such solutions, $e^{-\sigma_{i} t}$ is replaced by $e^{-\sigma_{i} r(x, y, z)}$ with $r(x, y, z)$ a real function of space variables. Therefore, both of the two solution forms of (1) have the same expression of phase relationship as $e^{i(\omega t-\alpha x-\beta y-\gamma z)}$. Consequently we only need to analyze the dispersion relation of (110).

Substituting (110) into Maxwell's equations (1) yields

$$
i\left(\omega M-\alpha K_{1}-\beta K_{2}-\gamma K_{3}\right)\left(\begin{array}{c}
\vec{H}_{0} \\
\vec{E}_{0}
\end{array}\right)=0
$$

where we have the same $M$ and $K_{i}$ as (23). This implies an exact dispersion relation given by

$$
\operatorname{det}\left(\omega M-\alpha K_{1}-\beta K_{2}-\gamma K_{3}\right)=0
$$

which reduces the exact dispersion relation as following

$$
\omega^{2}=\frac{1}{\varepsilon \mu}\left(\alpha^{2}+\beta^{2}+\gamma^{2}\right) .
$$

The discrete form of solution (110) is as

$$
\vec{H}_{j, l, m}^{k}=e^{-\sigma_{2} t_{k}} \vec{H}_{0} e^{i\left(\omega t_{k}-\alpha x_{j}-\beta y_{l}-\gamma u_{m}\right)}, \quad \vec{E}_{j, l, m}^{k}=e^{-\sigma_{1} t_{k}} \vec{E}_{0} e^{i\left(\omega t_{k}-\alpha x_{j}-\beta y_{l}-\gamma u_{m}\right)},
$$

where $j, l, m$ denote spatial indices, $k$ denotes temporal index. Substituting it into the Birkhoffian scheme (66) and the Birkhoffian box scheme (67), we obtain the same dispersion relation determinant as

$$
\operatorname{det}\left(\frac{\tan \left(\frac{\omega}{2} \tau\right)}{\tau} M-\frac{\tan \left(\frac{\alpha}{2} h_{x}\right)}{h_{x}} K_{1}-\frac{\tan \left(\frac{\beta}{2} h_{y}\right)}{h_{y}} K_{2}-\frac{\tan \left(\frac{\gamma}{2} h_{z}\right)}{h_{z}} K_{3}\right)=0,
$$

consequently we have the discrete dispersion relation as

$$
\omega^{2} \frac{\tan ^{2}\left(\frac{\omega \tau}{2}\right)}{\omega^{2} \tau^{2}}=\frac{1}{\varepsilon \mu}\left(\alpha^{2} \frac{\tan ^{2}\left(\frac{\alpha h_{x}}{2}\right)}{\alpha^{2} h_{x}^{2}}+\beta^{2} \frac{\tan ^{2}\left(\frac{\beta h_{y}}{2}\right)}{\beta^{2} h_{y}^{2}}+\gamma^{2} \frac{\tan ^{2}\left(\frac{\gamma h_{z}}{2}\right)}{\gamma^{2} h_{z}^{2}}\right) .
$$


Remark 4.3.1. Solutions of the Birkhoffian scheme (66) and the Birkhoffian box scheme (67) and the Hamiltonian box scheme (70) differ only by an attenuation factor with time, they have the same phase relation, therefore they have the same dispersion relation.

Remark 4.3.2. By the above Remark, the results for the Hamiltonian box scheme (70) also hold for the Birkhoffian scheme (66) and the Birkhoffian box scheme(67)(cf. [11, 12, 31, 32]):

1. The sign of the group velocity from the exact dispersion relation is preserved by the schemes.

2. The numerical dispersion relation has as many solution branches as the exact dispersion relation.

3. The quadrant in which the angle $\phi$ of exact group velocity belongs to, is preserved by the schemes.

\section{Numerical experiments}

\subsection{Numerical Verification Using Example with Exact Solutions}

In this section, we investigate the numerical behavior of the Birkhoffian scheme (66) and the Birkhoffian box scheme (67) discussed in Section 3. For comparison, the central box scheme (71) and the exponential FDTD method (72) are investigated too. The main work focuses on the preservation of the local/global conservation laws associated with Maxwell's equations. We will show some results for Maxwell's equations with all physical quantities independent of the third spacial variable $z$. We consider the two-dimensional (2D) Maxwell's equations for transverse-electric (TE) waves:

$$
\begin{aligned}
\frac{\partial \vec{E}_{x}}{\partial t} & =\frac{1}{\varepsilon} \frac{\partial \vec{H}_{z}}{\partial y}-\sigma_{1} \vec{E}_{x}, \\
\frac{\partial \vec{E}_{y}}{\partial t} & =-\frac{1}{\varepsilon} \frac{\partial \vec{H}_{z}}{\partial x}-\sigma_{1} \vec{E}_{y}, \\
\frac{\partial \vec{H}_{z}}{\partial t} & =\frac{1}{\mu}\left(\frac{\partial \vec{E}_{x}}{\partial y}-\frac{\partial \vec{E}_{y}}{\partial x}\right)-\sigma_{2} \vec{H}_{z} .
\end{aligned}
$$

Assume the system (117) has analytic solutions of the following form:

$$
Z=Z_{0}(t) \exp (i \omega t+i \alpha x+i \beta y)
$$


where $Z_{0}(t)$ denote $Z_{0} e^{-\frac{\sigma_{1}+\sigma_{2}}{2} t}$ which means an arbitrary constant vector $Z_{0}$ with attenuation in time direction. Assume the initial conditions of the problems are

$$
\begin{aligned}
\vec{E}_{x}(x, y, 0) & =-\frac{\sqrt{2}}{2} \cos (2 \pi x) \sin (2 \pi y), \\
\vec{E}_{y}(x, y, 0) & =\frac{\sqrt{2}}{2} \sin (2 \pi x) \cos (2 \pi y), \\
\vec{H}_{z}(z, y, 0) & =\sqrt{\frac{\varepsilon}{\mu}} \cos (2 \pi x) \cos (2 \pi y),
\end{aligned}
$$

then we have a set of exact solutions

$$
\begin{aligned}
\vec{E}_{x}(x, y, t) & =-\frac{\sqrt{2}}{2} e^{-\sigma_{1} t} \sin (2 \sqrt{2} t) \cos (2 \pi x) \sin (2 \pi y), \\
\vec{E}_{y}(x, y, t) & =\frac{\sqrt{2}}{2} e^{-\sigma_{1} t} \sin (2 \sqrt{2} t) \sin (2 \pi x) \cos (2 \pi y), \\
\vec{H}_{z}(z, y, t) & =\sqrt{\frac{\varepsilon}{\mu}} e^{-\sigma_{2} t} \cos (2 \sqrt{2} t) \cos (2 \pi x) \cos (2 \pi y) .
\end{aligned}
$$

In such case, the Birkhoffian scheme to (66) reduces to

$$
\begin{aligned}
\bar{\partial}_{t} R_{1} R_{2} \vec{E}_{x i, j}^{k} & =\frac{1}{\varepsilon} \partial_{y} \bar{R}_{t} R_{1} \vec{H}_{z i, j}^{k}-\frac{\sigma_{1}-\sigma_{2}}{2} \bar{R}_{t} R_{1} R_{2} \vec{E}_{x i, j}^{k} \\
\bar{\partial}_{t} R_{1} R_{2} \vec{E}_{y i, j}^{k} & =-\frac{1}{\varepsilon} \partial_{x} \bar{R}_{t} R_{2} \vec{H}_{z i, j}^{k}-\frac{\sigma_{1}-\sigma_{2}}{2} \bar{R}_{t} R_{1} R_{2} \vec{E}_{y i, j}^{k}, \\
\bar{\partial}_{t} R_{1} R_{2} \vec{H}_{z i, j}^{k} & =\frac{1}{\mu}\left(\partial_{y} \bar{R}_{t} R_{1} \vec{E}_{x i, j}^{k}-\partial_{x} \bar{R}_{t} R_{2} \vec{E}_{y i, j}^{k}\right)-\frac{\sigma_{1}-\sigma_{2}}{2} \bar{R}_{t} R_{1} R_{2} \vec{H}_{z i, j}^{k},
\end{aligned}
$$

and the Birkhoffian box scheme (67) reduces to

$$
\begin{aligned}
\tilde{\partial}_{t} R_{1} R_{2} \vec{E}_{x i, j}^{k} & =\frac{1}{\varepsilon_{1}\left(t_{k+\frac{1}{2}}\right)} \partial_{y} \hat{R}_{t} R_{1} \vec{H}_{z i, j}^{k}, \\
\tilde{\partial}_{t} R_{1} R_{2} \vec{E}_{y i, j}^{k} & =-\frac{1}{\varepsilon_{1}\left(t_{k+\frac{1}{2}}\right)} \partial_{x} \hat{R}_{t} R_{2} \vec{H}_{z i, j}^{k}, \\
\hat{\partial}_{t} R_{1} R_{2} \vec{H}_{z i, j}^{k} & =\frac{1}{\mu_{1}\left(t_{k+\frac{1}{2}}\right)}\left(\partial_{y} \tilde{R}_{t} R_{1} \vec{E}_{x i, j}^{k}-\partial_{x} \tilde{R}_{t} R_{2} \vec{E}_{y i, j}^{k}\right),
\end{aligned}
$$

where $\tilde{\partial}_{t} Z_{i, j}^{k}=\frac{e^{\sigma_{1} \tau} Z_{i, j}^{k+1}-Z_{i, j}^{k}}{\tau}, \tilde{R}_{t} Z_{i, j}^{k}=\frac{e^{\sigma_{1} \tau} Z_{i, j}^{k+1}+Z_{i, j}^{k}}{2}, \hat{\partial}_{t} Z_{i, j}^{k}=\frac{e^{\sigma_{2} \tau} Z_{i, j}^{k+1}-Z_{i, j}^{k}}{\tau}$ and $\hat{R}_{t} Z_{i, j}^{k}=$ $\frac{e^{\sigma_{2} \tau} Z_{i, j}^{k+1}+Z_{i, j}^{k}}{2}\left(\right.$ for $Z_{i, j}^{k}=\vec{E}_{x i j}^{k}, \vec{E}_{y i, j}^{k}, \vec{H}_{z i, j}^{k}$ ).

If $\sigma_{1}=\sigma_{2}$, we have the local conservation laws (93) and (94) which reduce to, respectively

- LQCL:

$$
\begin{aligned}
\frac{1}{2} \bar{\partial}_{t}\left[\varepsilon\left(\left|R_{1} R_{2} \vec{E}_{x i, j}^{k}\right|^{2}+\left|R_{1} R_{2} \vec{E}_{y i, j}^{k}\right|^{2}\right)+\mu\left|R_{1} R_{2} \vec{H}_{z i, j}^{k}\right|^{2}\right] & + \\
\partial_{x}\left(\bar{R}_{t} R_{2} \vec{H}_{z i, j}^{k} \bar{R}_{t} R_{2} \vec{E}_{y i, j}^{k}\right)-\partial_{y}\left(\bar{R}_{t} R_{1} \vec{H}_{z i, j}^{k} \bar{R}_{t} R_{1} \vec{E}_{x i, j}^{k}\right) & =0
\end{aligned}
$$


- LQD:

$$
\bar{\partial}_{t}\left[\frac{1}{\varepsilon}\left(\left|R_{1} \partial_{y} \vec{H}_{z i, j}^{k}\right|^{2}+\left|R_{2} \partial_{x} \vec{H}_{z i, j}^{k}\right|^{2}\right)+\frac{1}{\mu}\left(\left|R_{2} \partial_{x} \vec{E}_{y i, j}^{k}\right|^{2}+\left|R_{1} \partial_{y} \vec{E}_{x i, j}^{k}\right|^{2}\right)\right]=0,
$$

The global conservation laws (95) and (96) reduce to

- GQCL:

$$
h_{x} h_{y} \sum_{i, j} \bar{\partial}_{t}\left[\varepsilon\left(\left|R_{1} R_{2} \vec{E}_{x i, j}^{k}\right|^{2}+\left|R_{1} R_{2} \vec{E}_{y i, j}^{k}\right|^{2}\right)+\mu\left|R_{1} R_{2} \vec{H}_{z i, j}^{k}\right|^{2}\right]=0,
$$

\section{- GQD:}

$$
h_{x} h_{y} \sum_{i, j} \bar{\partial}_{t}\left[\frac{1}{\varepsilon}\left(\left|R_{1} \partial_{y} \vec{H}_{z i, j}^{k}\right|^{2}+\left|R_{2} \partial_{x} \vec{H}_{z i, j}^{k}\right|^{2}\right)+\frac{1}{\mu}\left(\left|R_{2} \partial_{x} \vec{E}_{y i, j}^{k}\right|^{2}+\left|R_{1} \partial_{y} \vec{E}_{x i, j}^{k}\right|^{2}\right)\right]=0(126)
$$

To verify the energy-preserving property of the schemes, we simulate the exact solutions (120) with $\varepsilon=\mu=1$ and $\sigma_{1}=\sigma_{2}=\bar{\sigma}=0.2$. We have also tried other parameter values and found that they make little difference in relatively behavior of different schemes. We compare the Birkhoffian scheme, the Birkhoffian box scheme, the central box scheme and the exponential FDTD scheme over the square domain $[0,1] \times[0,1]$. The time interval is from 0 to 20. The numerical errors of the discrete local and global conservation laws are calculated. In the presented figures, the computation parameters are always set as $h_{x}=h_{y}=0.1$ and $\tau=0.02$.

Fig. 1 shows the profiles of the magnetic component $\vec{H}_{z}$ at time $t=2$. It is hard to visually tell the difference between different schemes from these plots. So we plot the conservation error for each schemes. The maximum errors of the local conservations laws (123) and (124) are presented in Figs. 2 and 3 for the four schemes. The comparison of the errors of the global conservation laws (125) and (126) are presented in Figs. 4 and 5. These figures confirm that both local and global energies are preserved by the Birkhoffian and the Birkhoffian box schemes to machine round-off error. However both the popular exponential FDTD Yee's scheme and traditional multi-symplectic scheme (central box scheme) have relatively large error.

\subsection{Electromagnetic Pulse Propagation}

In this section, we solve the electromagnetic pulse (EMP) propagation problem using our numerical schemes. EMP interference is generally disruptive or damaging to electronic 

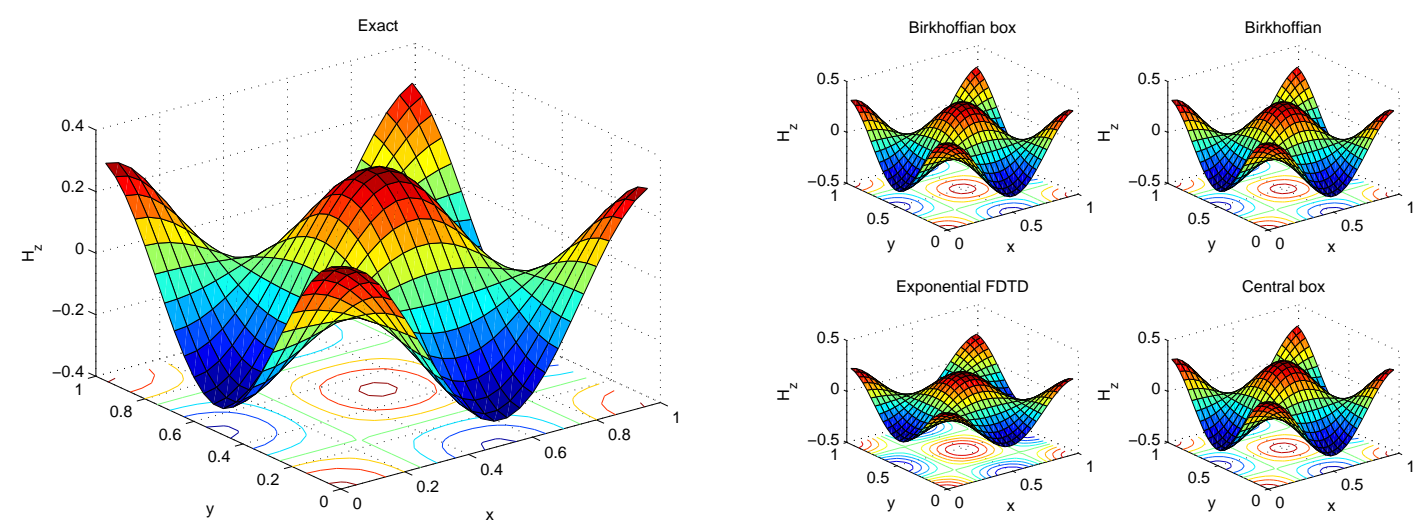

Figure 1: The profiles of $H_{z}$ with $\sigma_{1}=\sigma_{2}=0.2$ at time $t=2$.
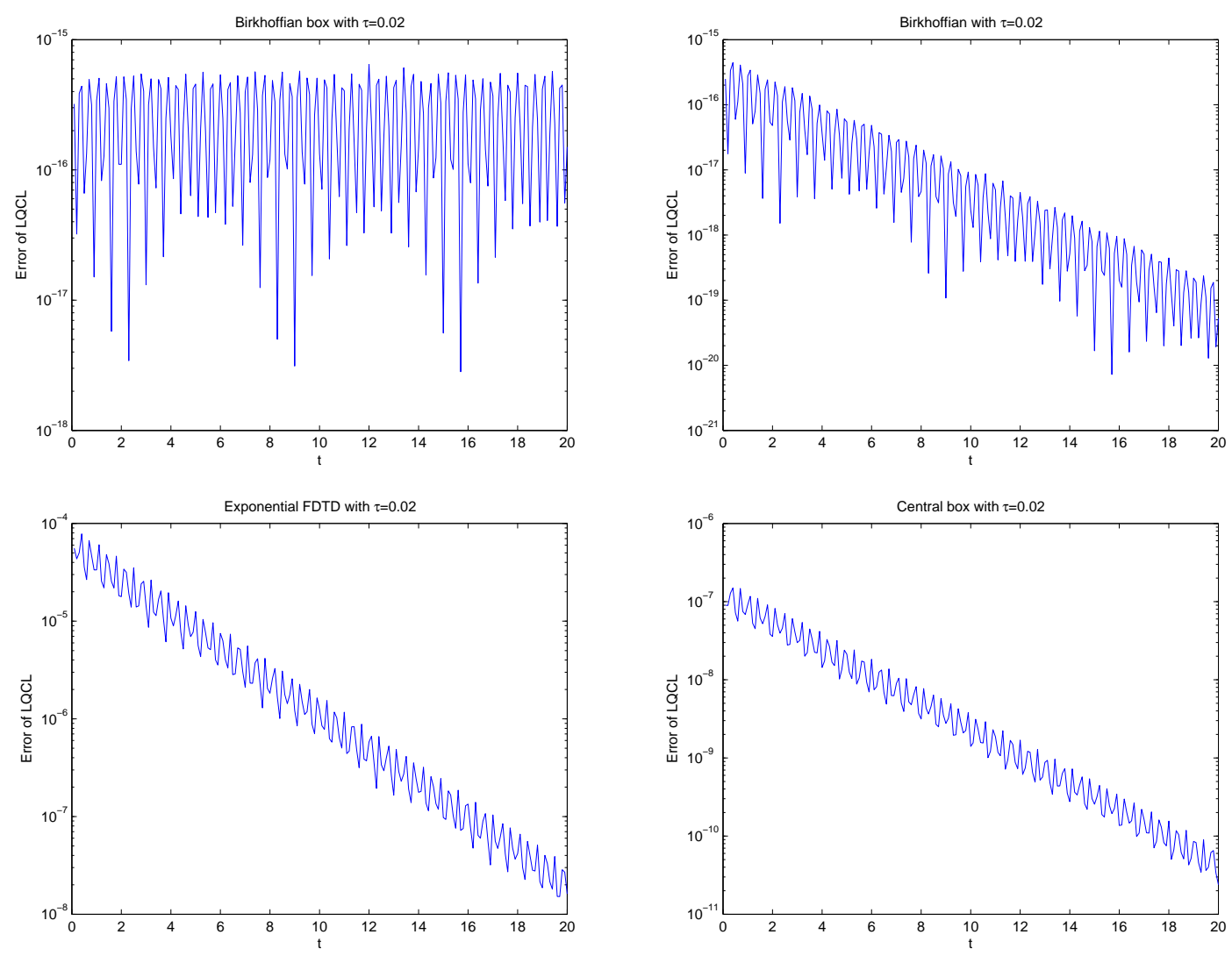

Figure 2: The max errors of the local conservation law (123). 

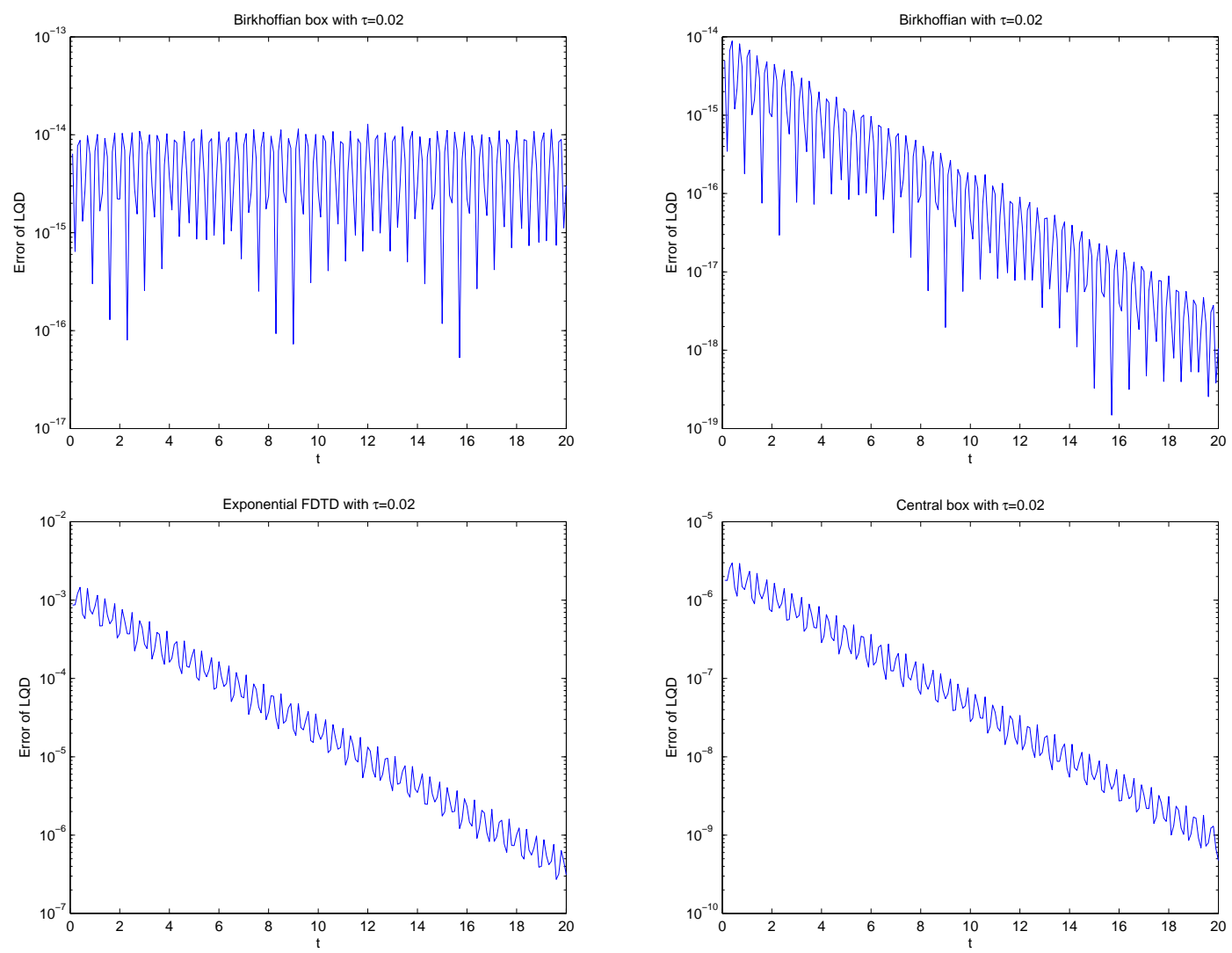

Figure 3: The max errors of the local conservation law (124). 

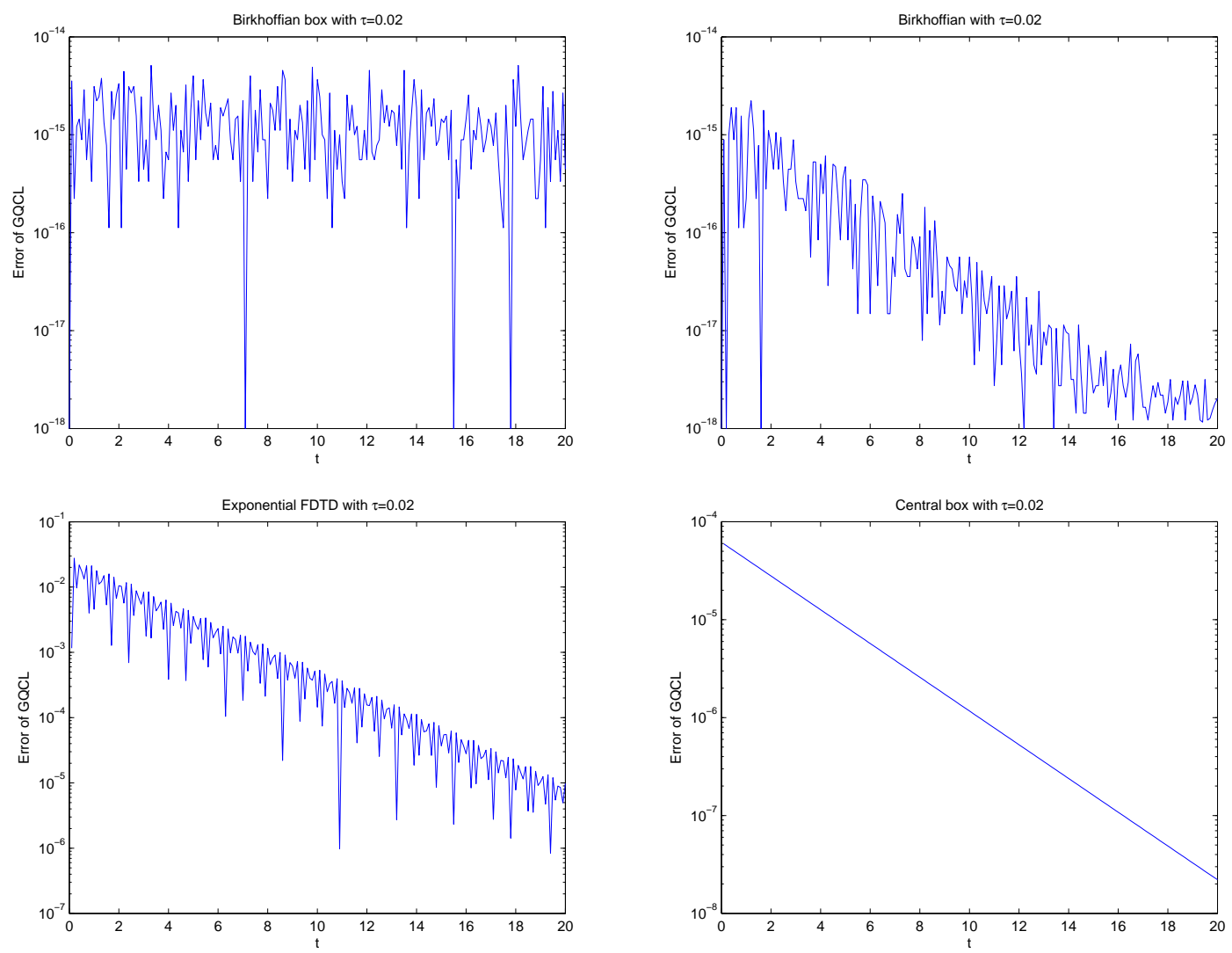

Figure 4: The errors of the global conservation law (125). 

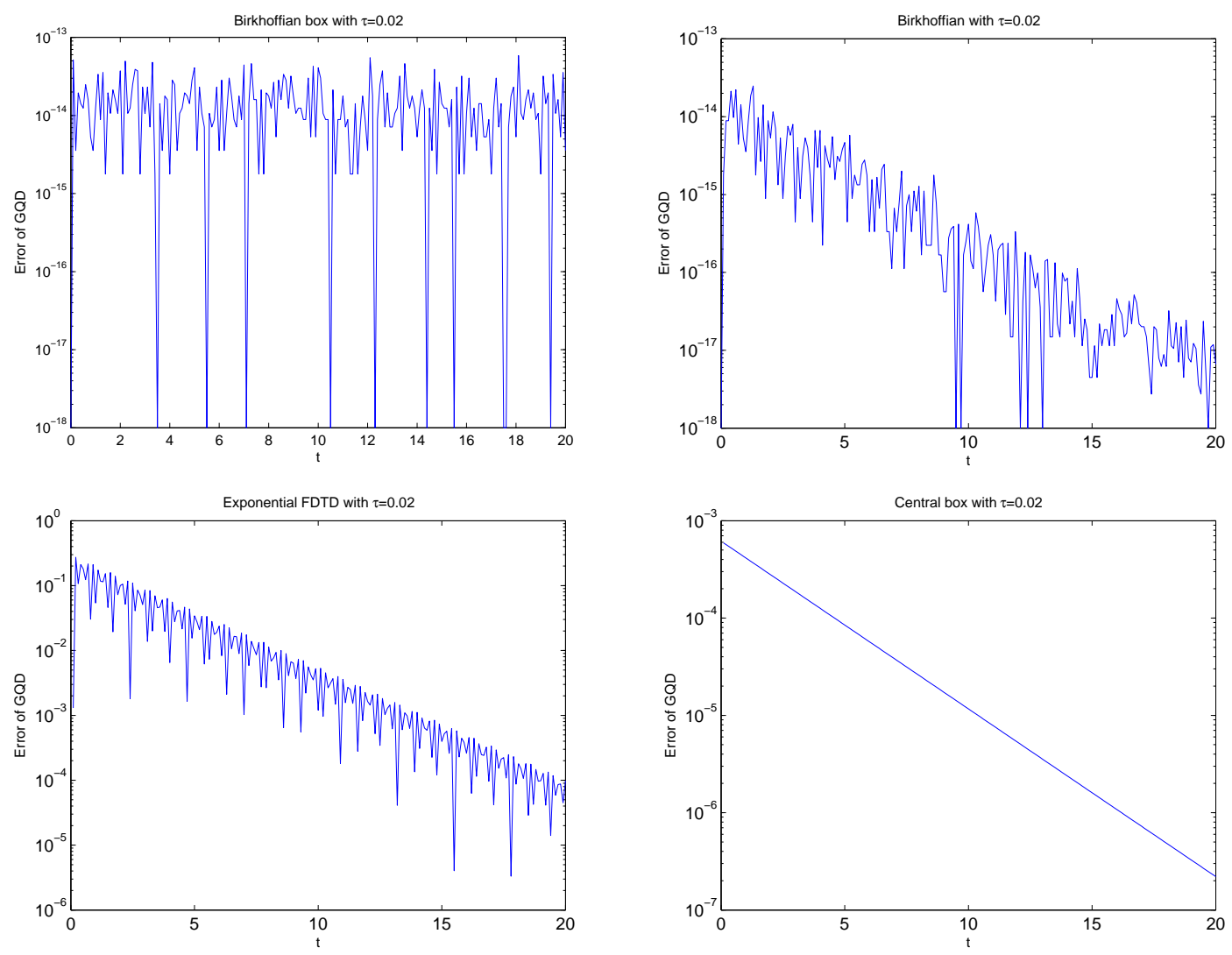

Figure 5: The errors of the global conservation law (126). 
equipment, and at higher energy levels a powerful EMP event such as a lightning strike can damage physical objects such as buildings and aircraft structures.

The current source (e.g., lightning strike) of our EMP problem locates in the sea at one kilometer from the seashore (land). We set up some receivers on the land to detect the electric-field of the EMP. From the received EMP wave-form, we can generally derive the magnitude and reaction history of the current source. Our computational domain (see Fig. (6) consists of three sub-domains that has three different materials (air, sea-water, and land) respectively. The electrical permittivity $(\varepsilon)$ of the air is taken as free-space permittivity $\varepsilon_{0}=8.854 \times 10^{-12}$ farads/meter. The relative permittivity of the land is $\varepsilon_{r}=10$., and for the sea-water it is $\varepsilon_{r}=80$.. The electric conductivity for the air, land, and sea-water is $\sigma_{\text {air }}=10^{-15}, \sigma_{\text {land }}=10^{-3}$, and $\sigma_{\text {sea }}=4.8$ with unit siemens $/$ meter. The land is 10 meters above the sea level (see Fig. (6). Although the computational domain is not exactly cylindrical symmetric, we solve this $3 \mathrm{D}$ problem as a $2 \mathrm{D}$ cylindrical problem for computational efficiency, and the results are compared well with real 3D simulations.

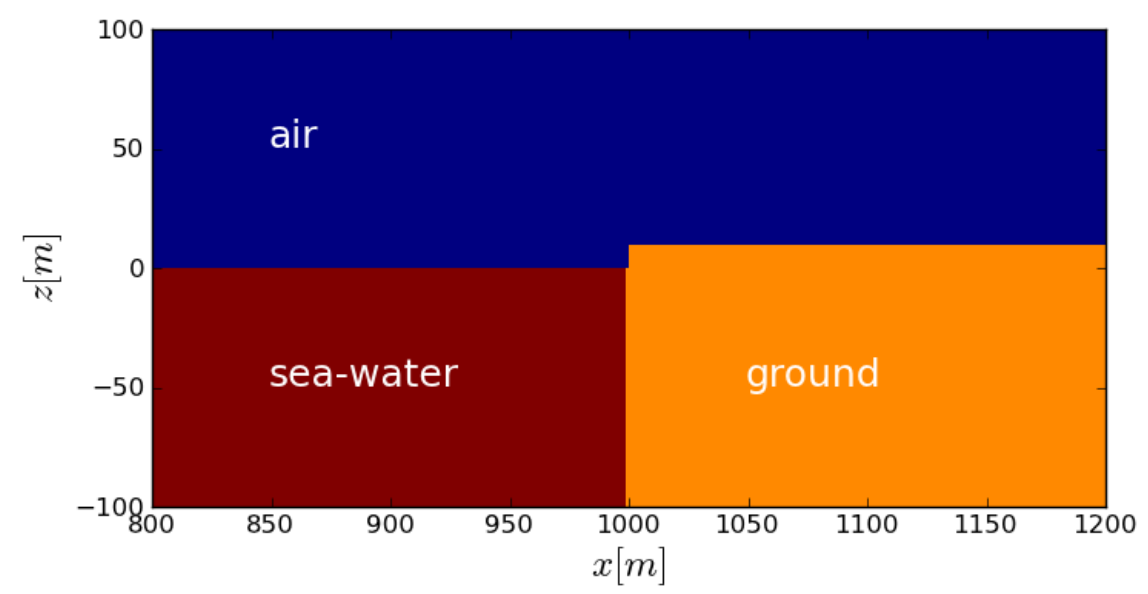

Figure 6: Part of physical domain for our EMP problem. The actual size of the domain is $r \times z=$ $[0 \mathrm{~m}, 10240 \mathrm{~m}] \times[-100 \mathrm{~m}, 1000 \mathrm{~m}]$. The electric conductivity of air, ground, and sea-water is $\sigma_{\text {air }}=10^{-15}$, $\sigma_{\text {lgrnd }}=10^{-3}$, and $\sigma_{\text {sea }}=4.8$. The relative permittivity of the air, ground, and sea-water is $\varepsilon_{r}^{a i r}=1$, $\varepsilon_{r}^{\text {grnd }}=10, \varepsilon_{r}^{\text {sea }}=80$. The current source locates at two meters above the sea level $(z=0)$ at $r=0$ axis

The physical domain is set up as $r \times z=[0 \mathrm{~m}, 10240 \mathrm{~m}] \times[-100 \mathrm{~m}, 1000 \mathrm{~m}]$ with $z=0$ as the sea-level. The current source locates at two meters above the sea level $(z=0)$ at $r=0$ axis. 


\subsubsection{Birkhoffian scheme for the cylindrical 2D TE mode}

We rewrite the 3D Maxwell's equation into 2D $\mathrm{TE}_{\phi}$ mode formulation

$$
\begin{aligned}
\varepsilon(r, z) \frac{\partial \vec{E}_{r}}{\partial t} & =-\frac{\partial \vec{H}_{y}}{\partial z}-\sigma(r, z) \vec{E}_{r}-\vec{J}_{r}, \\
\varepsilon(r, z) \frac{\partial \vec{E}_{z}}{\partial t} & =\frac{\partial \vec{H}_{y}}{\partial r}+\frac{\vec{H}_{y}}{r}-\sigma(r, z) \vec{E}_{z}-\vec{J}_{z}, \\
\mu(r, z) \frac{\partial \vec{H}_{y}}{\partial t} & =\frac{\partial \vec{E}_{z}}{\partial r}-\frac{\partial \vec{E}_{r}}{\partial z},
\end{aligned}
$$

where we have used $H_{y}$ as $H_{\phi}$. In what follows, $\tau$ is the temporal step-size, $h_{r}$ and $h_{z}$ are the step-sizes along $r$-direction and $z$-direction, respectively. $\vec{E}^{k}$ and $\vec{H}^{k}$ is the approximation of $\vec{E}(t, r, z)$ and $\vec{H}(t, r, z)$ at time $t_{k}, \bar{\sigma}=\frac{\sigma}{\varepsilon}$. From the 3D Birkhoffian scheme we can obtain the following semi-discrete Birkhoffian scheme for $2 \mathrm{D}$ cylindrical $\mathrm{TE}_{\phi}$ mode

$$
\begin{aligned}
e^{\frac{\bar{\sigma} \tau}{2}} \vec{E}_{r}^{k+1}-\vec{E}_{r}^{k}=\tau & {\left[-\frac{\bar{\sigma}}{4}\left(\vec{E}_{r}^{k}+e^{\frac{\bar{\sigma} \tau}{2}} \vec{E}_{r}^{k+1}\right)-\frac{1}{2 \varepsilon}\left(\frac{\partial \vec{H}_{y}^{k}}{\partial z}+e^{\frac{\bar{\sigma} \tau}{2}} \frac{\partial \vec{H}_{y}^{k+1}}{\partial z}\right)-\frac{1}{2 \varepsilon}\left(\vec{J}_{r}^{k}+e^{\frac{\bar{\sigma} \tau}{2}} \vec{J}_{r}^{k+1}\right)\right], } \\
e^{\frac{\bar{\sigma} \tau}{2}} \vec{E}_{z}^{k+1}-\vec{E}_{z}^{k}=\tau & {\left[-\frac{\bar{\sigma}}{4}\left(\vec{E}_{z}^{k}+e^{\frac{\bar{\sigma} \tau}{2}} \vec{E}_{z}^{k+1}\right)+\frac{1}{2 \varepsilon}\left(\frac{\partial \vec{H}_{y}^{k}}{\partial r}+e^{\frac{\bar{\sigma} \tau}{2}} \frac{\partial \vec{H}_{y}^{k+1}}{\partial r}\right)+\frac{1}{2 \varepsilon r}\left(\vec{H}_{y}^{k}+e^{\frac{\bar{\sigma} \tau}{2}} \vec{H}_{y}^{k+1}\right)\right.} \\
& \left.-\frac{1}{2 \varepsilon}\left(\vec{J}_{z}^{k}+e^{\frac{\bar{\sigma} \tau}{2}} \vec{J}_{z}^{k+1}\right)\right], \\
e^{\frac{\bar{\sigma} \tau}{2}} \vec{H}_{y}^{k+1}-\vec{H}_{y}^{k}= & \tau\left[\frac{\bar{\sigma}}{4}\left(\vec{H}_{y}^{k}+e^{\frac{\bar{\sigma} \tau}{2}} \vec{H}_{y}^{k+1}\right)+\frac{1}{2 \mu}\left(\left(\frac{\partial \vec{E}_{z}^{k}}{\partial r}+e^{\frac{\bar{\sigma} \tau}{2}} \frac{\partial \vec{E}_{z}^{k+1}}{\partial r}\right)-\left(\frac{\partial \vec{E}_{r}^{k}}{\partial z}+e^{\frac{\bar{\sigma} \tau}{2}} \frac{\partial \vec{E}_{r}^{k+1}}{\partial z}\right)\right)\right] .
\end{aligned}
$$

In our discretization, we denote a cell $(i, j)$ as $\left[r_{i-1 / 2}, r_{i+1 / 2}\right] \times\left[z_{i-1 / 2}, z_{i+1 / 2}\right]$, where $r_{i}=\left(i-\frac{1}{2}\right) h_{r}$, and $z_{j}=\left(j-\frac{1}{2}\right) h_{z}$. We use Yee's staggered grid to define the electric and magnetic field components: $\vec{E}_{r}^{k}(i, j)$ denotes the approximation of $\vec{E}_{r}(t, r, z)$ at $\left(t_{k}, r_{i}, z_{j-1 / 2}\right)$, $\vec{E}_{z}^{k}(i, j)$ denotes the approximation of $\vec{E}_{z}(t, r, z)$ at $\left(t_{k}, r_{i-1 / 2}, z_{j}\right)$, and $\vec{H}_{y}^{k}(i, j)$ denotes the approximation of $\vec{H}_{y}(t, r, z)$ at $\left(t_{k}, r_{i}, z_{j}\right)$. Combining Yee's central differencing with semidiscrete Birkhoffian scheme, we can obtain a fully-discretized Birkhoffian scheme.

We remark that at axis $r=0, \frac{H_{y}}{r}=\frac{\partial H_{y}}{\partial r}$ should be used to avoid computational overflow.

\subsubsection{Numerical set-up}

We use the grid size $h_{r}=h_{z}=1 m$, which results in a computational grid $10240 \times 1100$. We apply the reflective boundary condition at $r=0$ boundary, where $\vec{E}_{r}$ and $\vec{H}_{y}$ are antisymmetric and $\vec{E}_{z}$ are symmetric across $r=0$ axis. We apply the CPML [6] method at the other three boundaries with 10 PML layers. The initial values of $\vec{E}$ and $\vec{H}$ are zeros. 
To minimize the impact of the current source interaction with the PML layer, we impose a space cutoff to the current source. The current density $J$ can be none-zero only within $800 \mathrm{~m}$ radius of the source center, which is two meters above the sea level at $r=0$ axis. The current density $J$ has a wave-form of rational exponential equation:

$$
J(t)=\frac{A e^{\alpha\left(t-t_{0}\right)}}{1+\frac{\alpha}{\beta} e^{(\alpha+\beta)\left(t-t_{0}\right)}}
$$

where $\alpha$ is the exponential rise rate, and $\beta$ the exponential decay rate, $t_{0}$ is the time shift constant, and $A$ is the scaling factor related to the peak value. In all of our simulations, we take $A=10^{6}$ amperes $/ \mathrm{m}^{2}, t_{0}=300 \mathrm{~ns}, \alpha=10^{8} / \mathrm{s}=1 /$ shake, and $\beta=0.2 /$ shake, where 1 shake $=10^{-8} s=10 \mathrm{~ns}$. The current density in $r$ and $z$ direction is then specified as

$$
\begin{aligned}
& \vec{J}_{r}(r, z, t)=-\frac{J\left(t-\sqrt{r^{2}+z^{2}} / c\right)}{4 \pi\left(r^{2}+z^{2}\right)} \cdot \frac{r}{\sqrt{r^{2}+z^{2}}} \\
& \vec{J}_{z}(r, z, t)=-\frac{J\left(t-\sqrt{r^{2}+z^{2}} / c\right)}{4 \pi\left(r^{2}+z^{2}\right)} \cdot \frac{z}{\sqrt{r^{2}+z^{2}}}
\end{aligned}
$$

Fig. 7 shows the current density $\vec{J}_{z}$ history detected at 700 meters from the source center. The detector is 13 meters above the sea level $(z=0)$.
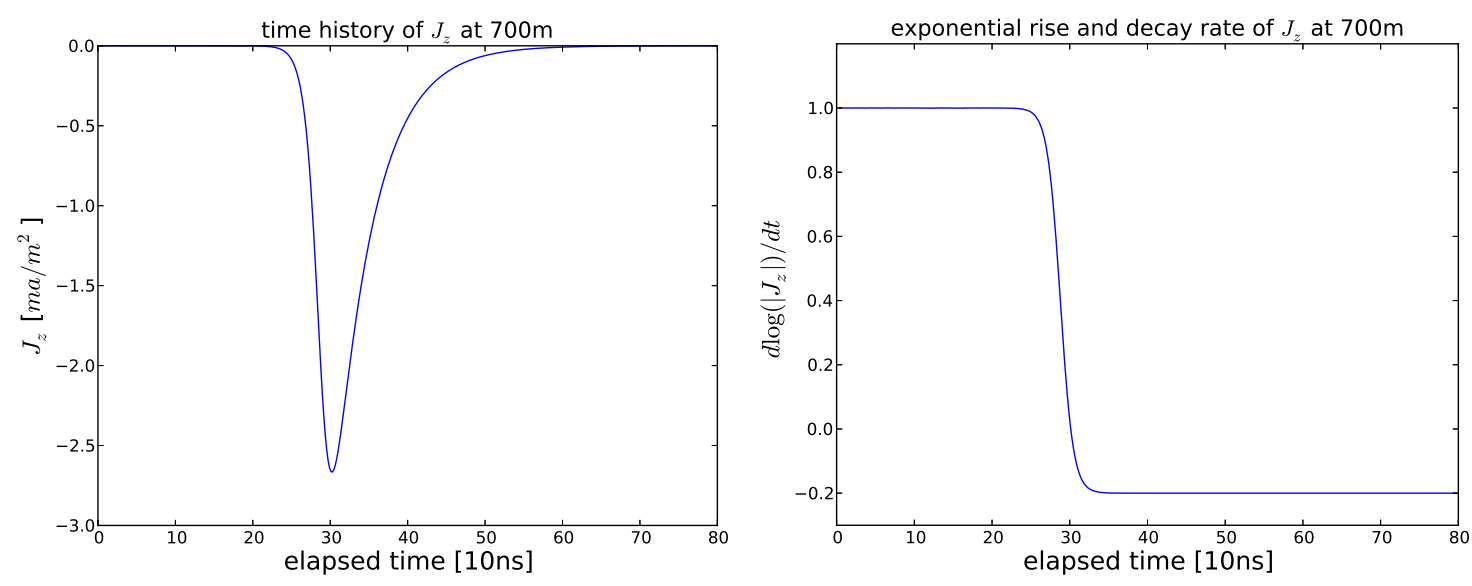

Figure 7: The current density $\vec{J}_{z}$ history detected at 700 meter from the source center. The right plot shows the magnitude of the exponential rise rate $(\alpha=1 /$ shake $)$ and decay rate $(\beta=0.2 /$ shake $)$ of the current source. It also shows the rising time is about 30 shakes (300 nano-seconds (ns)). This can also be recovered in $\vec{E}_{z}$ plot later.

We solve this problem use both the Birkhoffian scheme and traditional Yee's method plus the exponential integrator. We set up two receivers 10 meters above the ground: one is 1 kilometer from the source center, and the other is 7.3 kilometers from the source center. 

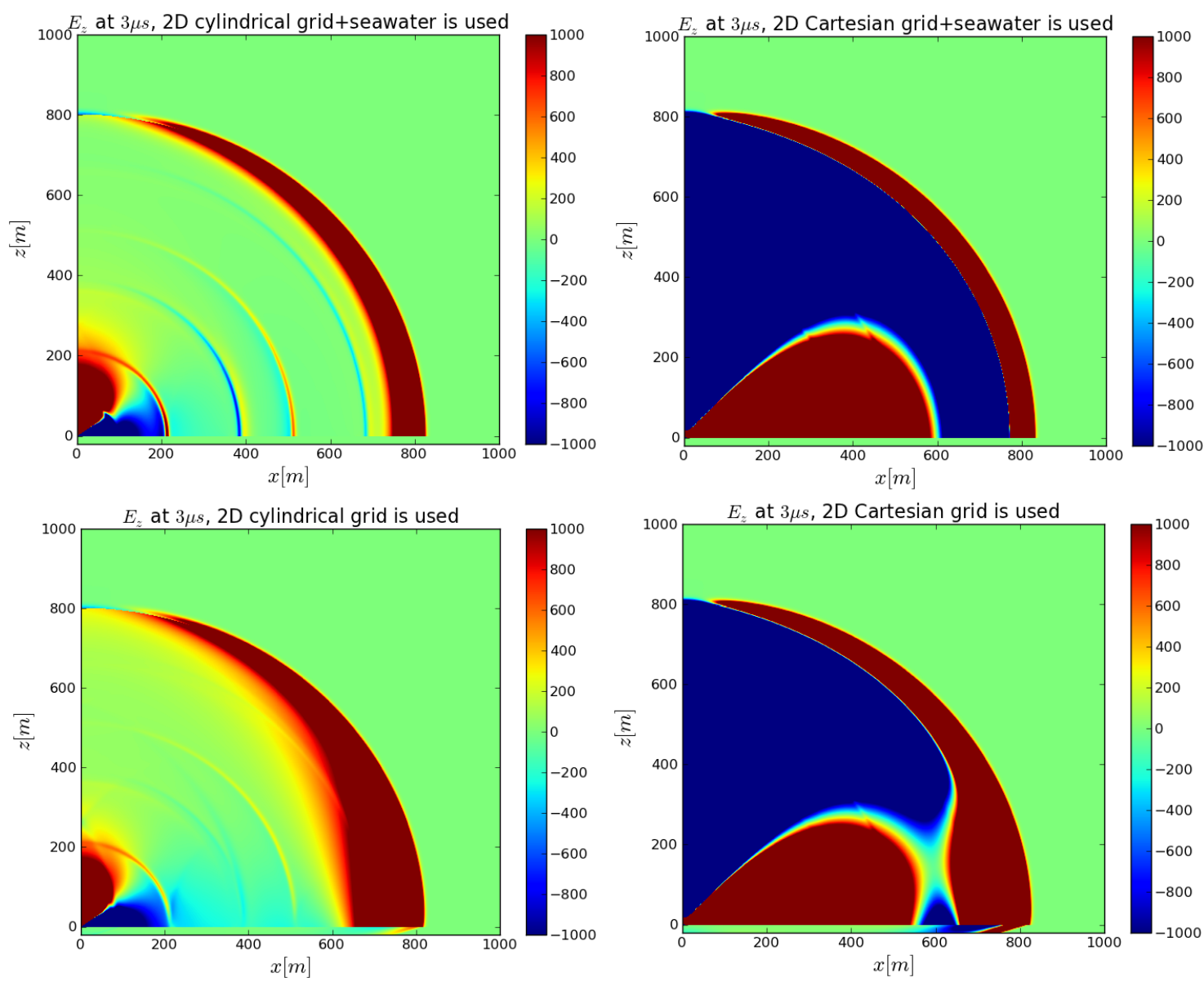

Figure 8: Comparison between different grids and different materials. The top two plots use the seawater v.s. the bottom two plots replace the sea-water with land. The left two plots use the cylindrical grid formulation v.s. the right two use the 2D Cartesian grid formulation. 
We first show the difference in results between the 2D cylindrical grid and 2D Cartesian grid. Although the only difference in the equation is the term $\vec{H}_{y} / r$ in $\vec{E}_{z}$ evolution, the magnitude and time evolution the electric and magnetic fields are dramatically different. Fig. 8 shows the comparison between different grids. We also provide result comparison between different materials. Because the sea-water has larger conductivity and permittivity than the ground, the corresponding electric field is also much larger (see Fig. 9).

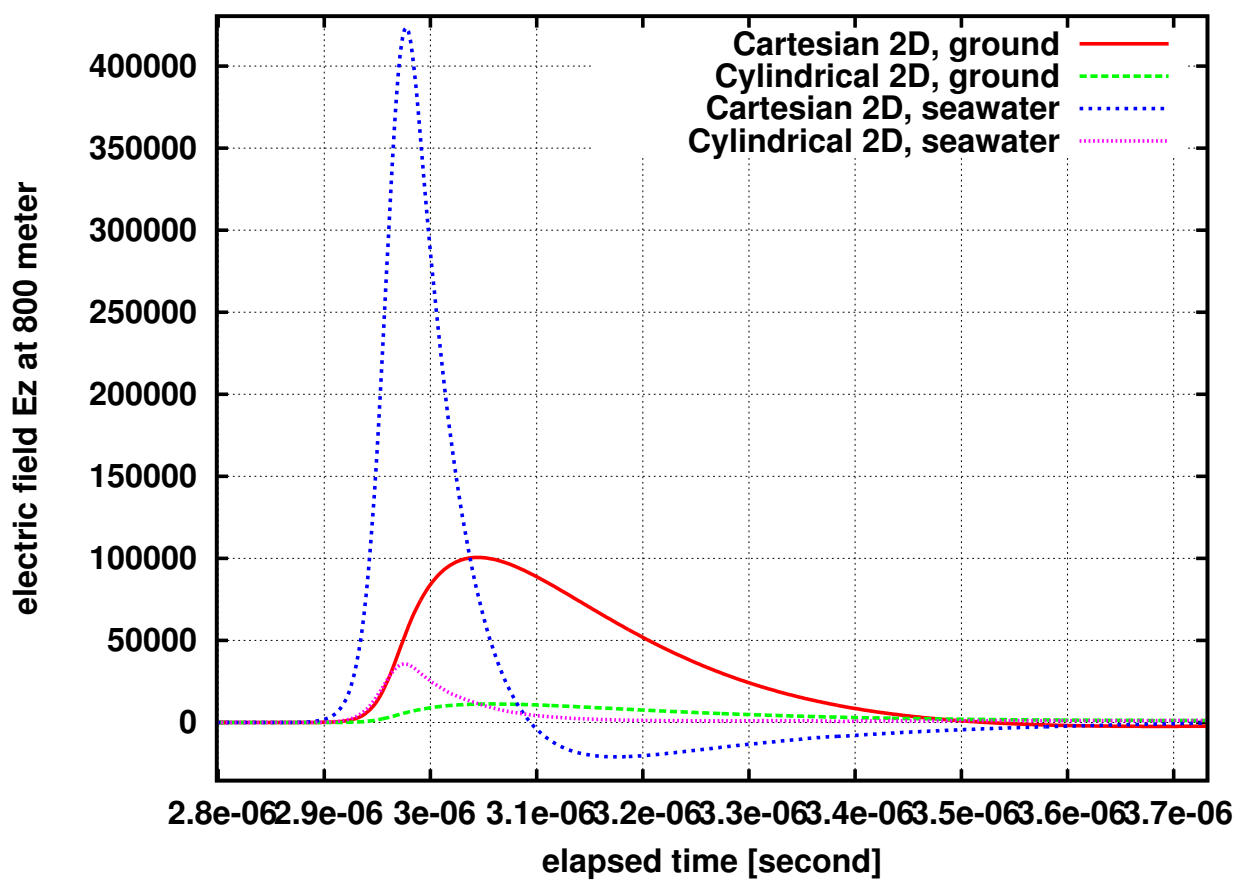

Figure 9: Comparison in $\vec{E}_{z}$ field between different grids and different materials used in the computation.

Next we show the difference in results between our Birkhoffian scheme and Yee's FDTD method. The cylindrical 2D and sea-water is used in these comparisons. The results are shown in Fig. 10.

\section{Conclusions and remarks}

For Maxwell's equations with dissipation terms in medium involving conductor loss, the non-autonomous and autonomous Birkhoffian formulations and the Hamiltonian formulation are discussed in this paper. Based on these variant formulations, three symplectic and multi-symplectic schemes (i.e. Birkhoffian scheme, Birkhoffian box scheme and Hamiltonian 

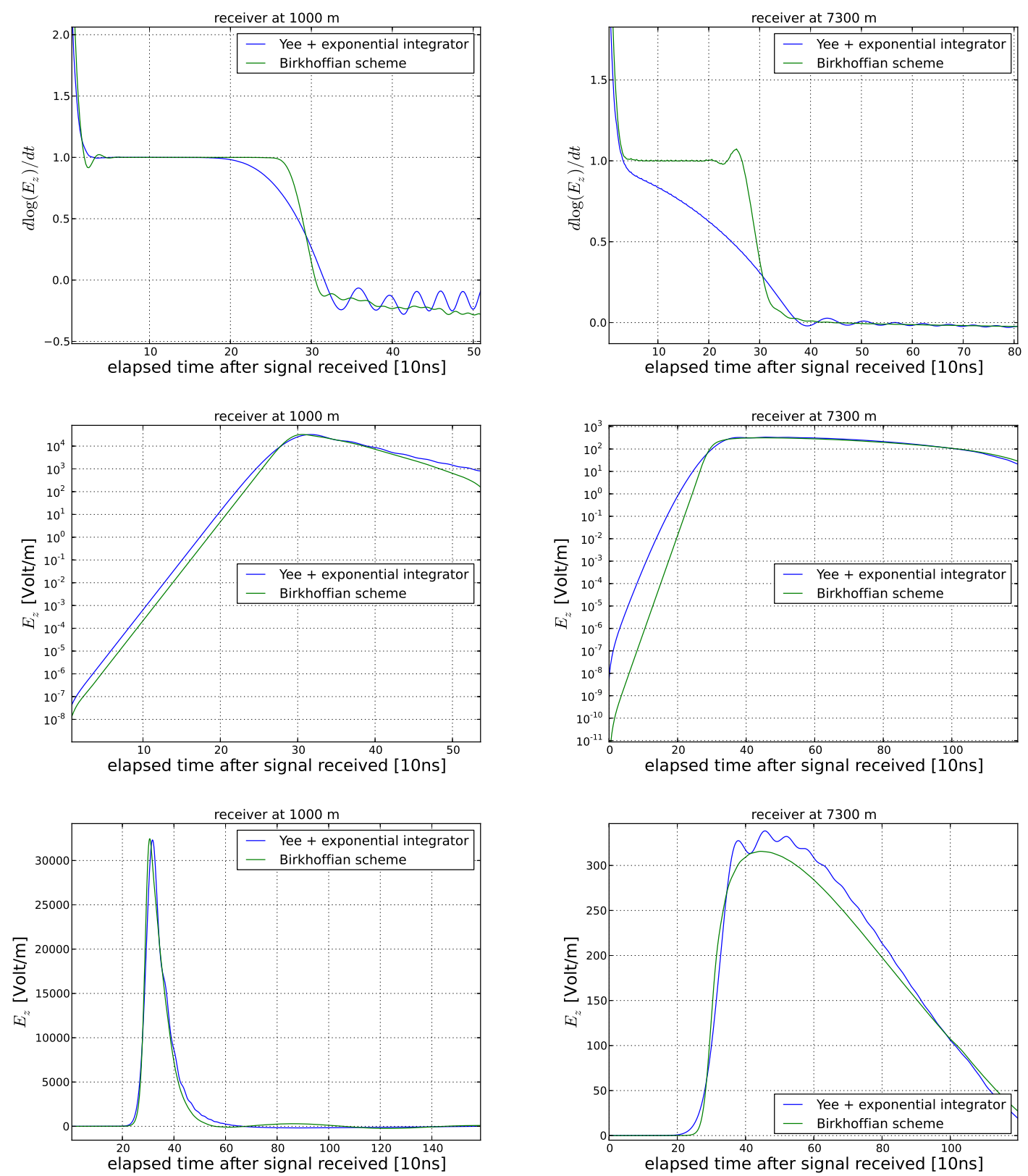

Figure 10: Numerical-result comparison between Birkhoffian scheme and Yee's FDTD scheme. The left column is for the receiver at 1000 meters and the right column is for the receiver at 7300 meters. Both receivers are located 13 meters above the sea-level. The top column plots the exponential rise rate of the electric field $\vec{E}_{z}$. The Birkhoffian scheme recovers the magnitude of the current source and reaction history very well even after long time propagation. 
box scheme) are proposed. All the proposed schemes are proved to satisfy the conservation of energy: quadratic-conservation property and multi-symplectic energy/momentum conservation. Moreover, we show that the schemes inherit the dispersion relation of the central box scheme applied to the Maxwell's equation without conductor loss. It implies that the numerical dispersion of the proposed schemes contains no non-physical solution branches. In particular, the schemes are strictly proved to be unconditionally stable and dissipation-preserving for Maxwell's equations in PML medium (i.e. $\sigma_{1}=\sigma_{2}$ ).

The numerical experiments of the Birkhoffian scheme and the Birkhoffian box scheme are compared with other methods (i.e. the central box scheme and the exponential FDTD scheme ) used frequently to solve Maxwell's equations. The comparison shows that the proposed schemes have a clear superiority over the other schemes in preserving the local and global conservation laws. The Birkhoffian scheme also shows its advantage in solving large scale and long time application problems.

In the numerical comparison, the Birkhoffian scheme not only preserves the conservation laws but also preserves the dissipation of the energy. The Birkhoffian box scheme only preserves the conservation laws, but does not figure out the dissipation. It is pointed in section 3 that the two proposed schemes are equivalent for Maxwell's equations in PML medium. But the equivalent schemes do not produce a same numerical results, as shown in the numerical comparison. The reason for this phenomenon is that the schemes are developed based on different formulations. It is well-known that different formulations for the same physical law can lead to different computational techniques in solving the same problems. Therefore, the study of the structures for PDEs is necessary.

Acknowledgments: Research of H. Su is supported by NNSFC Program project 10701081 and 11071251. Research of S. Li is supported by the Laboratory Directed Research and Development (LDRD) Program at Los Alamos National Laboratory under the auspices of the Department of Energy.

\section{References}

[1] Jean-Pierre Berenger, A perfectly matched layer for the absorption of electromagnetic waves, J. Comput. Phys. 114 (1994) 185-200.

[2] K.S. Yee, Numerical solution of initial boundary value problems involving Maxwell's equations in isotropic media, IEEE Trans. Antennas and Propagation, 14(1961) 302-307. 
[3] P. Monk, An analysis of Nedele's method for spatial discretization of Maxwell's equations, J. Comput. Appl. Math., 47(1993) 101-121.

[4] P. Monk, E. Süli, A convergence analysis of Yee's scheme on nonuniform grids, SIAM J. Numer. Anal. 31(1994). 393-412.

[5] A. Taflove, M.E. Brodwin, Numerical solution of steady-state electromagnetic scattering problems using the time-dependent Maxwell's equations, IEEE. Trans. Microw. Theory Tech., 23(1975) 623-630.

[6] A. Taflove, S. Hagness, Computational electromagnetics: The finite-difference time-domain method, 3rd ed., Artech House, Boston, 2005.

[7] Jialin Hong, Hongyu Liu, Geng Sun, The multi-symplecticity of partitioned Runge-Kutta method for Hamiltonian PDEs, Math. Comput. 75 (2005) 167-181.

[8] Yajuan Sun, Quadratic inviriants and multi-symplecticity of partitioned Runge-Kutta methods for Hamiltonian PDEs, Numer. Math. 106 (2007) 691-715.

[9] Hongling Su, Mengzhao Qin, R. Scherer, A multisymplectci geometry and a multisymplectic scheme for Maxwell's equations, Int. J. Pure Appl. Math. 34 (2007) 1-17.

[10] L. Kong, J. Hong, J. Zhang, Splitting multisymplectic integrators for Maxwell's equations, J. Comput. Phys. 229 (2010) 4259-4278.

[11] Y. Sun, P.S.P. Tse, Symplectic and multisymplectic numerical methods for Maxwell's equations, J. Comput. Phys. 230 (2011) 2076-2094.

[12] W. Cai, Y. Wang, Y. Song, Numerical dispersion analysis of a multi-symplectic scheme for the three dimensional Maxwell's equations, J. Comput. Phys. 234 (2013) 330-352.

[13] Hongling Su, Mengzhao Qin, Yushun Wang, R. Scherer, Multi-symplectic Birkhoffian structure for PDEs with dissipation terms, Phys. Lett. A 374 (2010) 2410-2416.

[14] N. Anderson, A.M. Arthurs, Helicity and variational principles for Maxwell's equations, INT. J. Electronics 54 (1983) 861-864.

[15] T.J. Bridges, S. Reich, Multi-symplectic integrators: numerical schemes for Hamiltonian PDEs that conserve symplecticity, Phys. Lett., A 284 (2001) 184-193.

[16] J.E. Marsden, G. W. Patrick \& S. Shkoller, Multisymplectic gometry, variational integrators, and nonlinear PDEs. Commun. Math. Phys. 199 (1998) 351-395.

[17] S. Reich, Multi-symplectic Runge-Kutta collocation methods for Hamiltonian wave equations, J. Comput. Phys. 157 (2000) 473-499.

[18] W.B. Chen, X.J. Li, D. Liang, Energy-conserved splitting FDTD methods for Maxwell's equations, Numer. Math. 108 (2008) 445-485.

[19] W.B. Chen, X.J. Li, D. Liang, Energy-conserved splitting finite-difference time-domain methods for Maxwell's equations in three dimensions, SIAM J. Numer. Anal. 48 (2010) 1530-1554.

[20] T.J. Bridges, Multi-symplectic structures and wave propagation, Math. Proc. Camb. Phil. Soc. 121 (1997) 147-190.

[21] J. Hong, C. Li, Multi-symplectic Runge-Kutta methods for nonlinear Dirac equations, J. Comput. Phys. 211 (2006) 448-472.

[22] T.J. Bridges, A geometrical formulation of the conservation of wave action and its implications for signature and the classification of instabilities, Proc. Roy. Soc. Lond. A 453 (1997) 1365-1395. 
[23] T. Hirono, W.W. Lui, K. Yokoyama, Time-domain simulation of electromagnetic field using a symplectic integrator, IEEE Microw. Guided Wave Lett. 7 (1997) 279-281.

[24] Zhi-Xiang Huang, Xian-Liang Wu, Symplectic partitioned Runge-Kutta scheme for Maxwell's equations, Inter. J. Quant. Chem. 106 (2006) 839-842.

[25] Hongling Su, Shengtai Li, Structure-preserving numerical methods for infinite-dimensional Birkhoffian systems, J. Sci. Comput. 65 (2015) 196-223.

[26] Feng Kang, Qin Mengzhao, Symplectic Geometric Algorithms for Hamiltonian Systems, Springer, Heidelberg (2009).

[27] Z. Shang, Generating functions for volume-preserving mappings and Hamilton-Jacobi equations for source-free systems, Sci. China (Ser. A) 37 (1994) 1172-1188.

[28] SU Hong-Ling, QIN Meng-Zhao, Symplectic schemes for Birkhoffian system, Commun. Theor. Phys. 41 (2004) 329-334.

[29] John C. Strikwerda, Finite difference schemes and partial differential equations, International Thomson Publishing, Chapman \& Hall, New York, 1989, p.103.

[30] J.B. Schneider, C.L. Wagner, FDTD dispersion revisited: faster-than-light propagation, IEEE Microw. Guided Wave Lett., 9 (1999) 54-56.

[31] L.N. Trefethen, Group velocity in finite difference schemes, SIAM Rev. 24 (1982), 113-136.

[32] J. Frank, B.E. Moore, S. Reich, Linear PDEs and numerical methods that preserve a multisymplectic conservation law, SIAM J. Sci. Comput. 28 (2006) 260-277. 